\title{
Magnetic Doppler imaging of 53 Camelopardalis in all four Stokes parameters ${ }^{\star}$
}

\author{
O. Kochukhov ${ }^{1, \star \star}$, S. Bagnulo ${ }^{2}$, G. A. Wade ${ }^{3}$, L. Sangalli ${ }^{3}$, N. Piskunov ${ }^{1}$, J. D. Landstreet ${ }^{4}$, \\ P. Petit ${ }^{5}$, and T. A. A. Sigut ${ }^{4}$
}

1 Uppsala Astronomical Observatory, Box 515, 75120 Uppsala, Sweden

2 European Southern Observatory, Casilla 19001, Santiago 19, Chile

${ }^{3}$ Department of Physics, Royal Military College of Canada, Box 17000, Kingston, Ontario, K7K 4B4 Canada

${ }^{4}$ Physics and Astronomy Department, University of Western Ontario, London, Ontario, N6A 3K7 Canada

5 Observatoire Midi-Pyrénées, 14 avenue Édouard Belin, 31400 Toulouse, France

Received 30 June 2003 / Accepted 10 October 2003

\begin{abstract}
We present the first investigation of the structure of the stellar surface magnetic field using line profiles in all four Stokes parameters. We extract the information about the magnetic field geometry and abundance distributions of the chemically peculiar star 53 Cam by modelling time-series of high-resolution spectropolarimetric observations with the help of a new magnetic Doppler imaging code. This combination of the unique four Stokes parameter data and state-of-the-art magnetic imaging technique makes it possible to infer the stellar magnetic field topology directly from the rotational variability of the Stokes spectra. In the magnetic imaging of 53 Cam we discard the traditional multipolar assumptions about the structure of magnetic fields in Ap stars and explore the stellar magnetic topology without introducing any global a priori constraints on the field structure. The complex magnetic model of 53 Cam derived with our magnetic Doppler imaging method achieves a good fit to the observed intensity, circular and linear polarization profiles of strong magnetically sensitive Fe II spectral lines. Such an agreement between observations and model predictions was not possible with any earlier multipolar magnetic models, based on modelling Stokes $I$ spectra and fitting surface averaged magnetic observables (e.g., longitudinal field, magnetic field modulus, etc.). Furthermore, we demonstrate that even the direct inversion of the four Stokes parameters of 53 Cam assuming a loworder multipolar magnetic geometry is incapable of achieving an adequate fit to our spectropolarimetric observations. Thus, as a main result of our investigation, we discover that the magnetic field topology of $53 \mathrm{Cam}$ is considerably more complex than any low-order multipolar expansion, raising a general question about the validity of the multipolar assumption in the studies of magnetic field structures of Ap stars. In addition to the analysis of the magnetic field of 53 Cam, we reconstruct surface abundance distributions of $\mathrm{Si}, \mathrm{Ca}, \mathrm{Ti}, \mathrm{Fe}$ and $\mathrm{Nd}$. These abundance maps confirm results of the previous studies of $53 \mathrm{Cam}$, in particular dramatic antiphase variation of $\mathrm{Ca}$ and $\mathrm{Ti}$ abundances.
\end{abstract}

Key words. stars: chemically peculiar - stars: magnetic fields - polarization - stars: individual: 53 Cam

\section{Introduction}

The magnetic field plays a fundamental role in the physics of the atmospheres of many different stars in the H-R diagram. Presumably in a large fraction of late-type stars, the magnetic field is responsible for phenomena that are well observed in the sun, such as, e.g., heating the corona, driving stellar winds, producing stellar flares. In late-type stars, the magnetic field is not globally organized, but appears to be concentrated primarily in limited regions (spots) of opposite polarity that form, migrate,

Send offprint requests to: O. Kochukhov, e-mail: oleg.kochukhov@astro.uu.se

* Based on observations obtained with the Bernard Lyot telescope of the Pic du Midi Observatory and Isaac Newton Telescope of the La Palma Observatory.

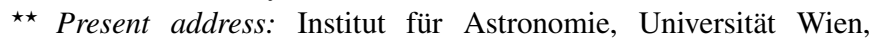
Türkenschanzstraße 17, 1180 Wien, Austria. evolve and disappear on a usually short time scale. However, except in the sun, a magnetic field structured on small scales is difficult to detect through spectropolarimetric techniques, since in a stellar (hence disk-integrated) observation, the polarization signal coming from surface regions characterized by opposite field polarity tends to cancel out.

The magnetic fields of early-type stars have quite different characteristics (and probably a different origin) than those of late-type stars. In early-type stars, the surface magnetic field is static on a timescale of at least many decades, and appears to be "frozen" into a rigidly rotating atmosphere. The magnetic field is organized on a large scale, permeating the entire stellar surface, with a relatively high field strength (typically of a few hundreds up to a few tens of thousands of Gauss). The presence of a magnetic field occurs only in those stars in which the photospheric abundances of several chemical 
elements - mostly silicon, iron-peak and rare-earth elements are largely different than in the solar case. These are the magnetic chemically peculiar stars of the upper main sequence, or Ap stars, that exhibit a wealth of characteristics that are not observed in other "normal" stars or non-magnetic metallic-line and mercury-manganese peculiar stars located in the same region of the H-R diagram (see, e.g., Landstreet 1992). Due to its global nature, the magnetic field of Ap stars can be much more easily detected and studied than that of late-type stars. Therefore, Ap stars represent a primary target for spectropolarimetric observations, and for the development and testing of the instruments and modelling techniques for studies of stellar magnetic fields.

The reason why certain (some 10-15\%) upper main sequence stars are magnetic and chemically peculiar, and why the remaining ones are "normal" stars, is a mystery. One of the key and basic problems to be solved is, in fact, the origin of the magnetic field itself - see Moss (2001) for a recent review on this theme. Another interesting yet not well understood phenomenon is the interplay between magnetic field and geographic distribution of the chemical elements over the stellar surface. The study of this kind of phenomenon is the scope of the diffusion theory. Introduced by Michaud (1970), diffusion theory explains the presence of chemically peculiarities in upper main sequence stars as the result of a competition between radiative pressure and gravitational settling. If present, a magnetic field influences the distribution of chemical elements see Alecian \& Stift (2002) for a study of how the radiative accelerations experienced by chemical elements in a stellar atmosphere depend on field strength and orientation. Thus, we expect that magnetic field topology and geographic distribution of chemical elements are correlated. For instance, certain elements may concentrate more in regions characterized by a radial field rather than in regions where the vector field is parallel to the stellar surface. A priori, the distribution of chemical elements may also depend on the local magnetic field modulus. As a matter of fact, we are not yet capable of doing precise predictions: assuming a certain model atmosphere, and a given magnetic morphology, only few schematic suggestions can be made on how (some) elements diffuse in the stellar atmosphere (e.g., Michaud et al. 1981). These simple models generally fail to account for the observed abundance distributions and cannot fully explain the enormous star-to-star variations of the geometry and magnitude of the surface chemical inhomogeneities.

Links between magnetic field topology and element distribution are also sought through analysis of observations. The earliest investigations (e.g., Stibbs 1950; Preston 1972), were limited to studying the correlation of the line strength variability for a few chemical elements with the mean longitudinal field variation. Explicit attempts to recover magnetic field topology and element distribution in individual stars have been made, for instance, by Landstreet (1988) and Landstreet et al. (1989), and, more recently, by Khokhlova et al. (2000). However, it should be noted that most of the existing works assume that the star's magnetic morphology is relatively simple (e.g., axisymmetric), whereas recently (see, e.g., Leroy et al. 1995; Bagnulo et al. 1999) it has been convincingly argued that the magnetic morphology of Ap and Bp stars is in fact fairly complex, and that in many cases cannot be described in terms of pure dipolar topologies or axisymmetric superpositions of dipole and quadrupole components. Furthermore, recently Bagnulo et al. (2001) have suggested that even general non-axisymmetric low-order multipolar models may not provide an adequate approximation of the actual magnetic topologies in some Ap stars and that the magnetic field may be structured on much smaller scales than was previously thought.

New advances in the quality and quantity of the available observational data have been recently obtained thanks to an extensive observational campaign with the MuSiCoS spectropolarimeter at the 2-m Telescope Bernard Lyot of the Pic du Midi Observatory (Wade et al. 2000a). Complete time series of Stokes IQUV profiles for a number of bright magnetic chemically peculiar stars have been collected in the last few years, opening the possibility to study magnetic stars with unprecedented observational constraint. At the same time, Piskunov \& Kochukhov (2002) and Kochukhov \& Piskunov (2002) have completed the development of a computer code for Doppler imaging of magnetic stars that, based on no a priori model parameterization, performs the inversion of Stokes IQUV profiles, and recovers the star's vector magnetic field morphology and element distribution. The availability of these new data and modelling tools now makes it possible to do a much more meaningful analysis of the magnetic morphology and element distribution than in the past. As a final goal, the reconstruction of the magnetic field and the surface abundance distributions of the chemical elements for a significant number of chemically peculiar stars will allow us to set constraints to the diffusion theory, and to better correlate the characteristics of the magnetic field with other fundamental stellar parameters. Altogether, a clearer picture of the magnetic morphologies of chemically peculiar stars may give a crucial contribution to understanding the problem of the origin of the magnetic field in early-type stars.

Along the lines of the strategy discussed above, here we present an exhaustive study of a famous and well-observed magnetic star, 53 Camelopardalis. Our investigation is utterly unique, by the virtue of the fact that we use the first highresolution four Stokes parameter stellar spectroscopic observations, and that these data are interpreted using a sophisticated magnetic Doppler imaging code incorporating detailed spectrum synthesis calculations of the Stokes $I Q U V$ profiles.

This paper is organized as follows. In Sect. 2 we describe the observations used in this work. In Sect. 3 we derive the stellar fundamental parameters. In Sect. 4 we review the previous strategies adopted for studying stellar magnetic field morphologies. In Sect. 5 we derive simultaneously a model for the magnetic field of $53 \mathrm{Cam}$ and for the Fe abundance distribution. Assuming the magnetic model of Sect. 5, in Sect. 6 we derive the distribution of other chemical elements ( $\mathrm{Si}, \mathrm{Ca}, \mathrm{Ti}$ and $\mathrm{Nd}$ ). In Sect. 7 we summarize and discuss the results of this work.

\section{Observational data}

\subsection{Spectropolarimetric observations}

Polarized spectra of 53 Cam were obtained during 1997, 1998 and 2000 using the MuSiCoS spectropolarimeter, connected 
to the 2-m Bernard Lyot Telescope (TBL) at Pic du Midi observatory.

The MuSiCoS instrument consists of a table-top crossdispersed échelle spectrograph, fed via a double optical fibre directly from a Cassegrain-mounted dedicated polarization analysis module. In one single exposure, this apparatus allows the acquisition of a stellar spectrum in a given polarization state (Stokes $V, Q$ or $U$ ) throughout the spectral range 450 to $660 \mathrm{~nm}$ with a resolving power of about 35000 . The optical characteristics of the spectrograph and polarization analyser, as well as the spectropolarimeter observing procedures, are described in detail by Baudrand \& Böhm (1992) and by Donati et al. (1999).

In normal operation, starlight enters the analyser at the Cassegrain focus. The beam then may optionally pass through a rotatable $\lambda / 4$ retarder (in the case of Stokes $V$ observations) or not (in the case of Stokes $Q$ or $U$ observations). The beam then intersects a Savart-type beamsplitter which separates the stellar light into two beams which are respectively polarized along and perpendicular to the instrumental reference azimuth. The analysed beams are then focally reduced to an aperture of $f / 2.5$ for injection into the double $50 \mu \mathrm{m}$ fibre, which transports the light to the spectrograph. Spectra in both orthogonal polarizations are thereby recorded simultaneously by the thinned $1024 \times 1024$ pixel SITE CCD detector.

The instrument is calibrated mechanically (or tuned) in the manner described by Donati et al. (1999). This three-step procedure (alignment of the polarizing beamsplitter with the instrument axis, alignment of the wave plates with respect to the beamsplitter, and alignment of the fibres and the beamsplitter) ensures the accuracy of the polarization analysis. This procedure was performed during each re-mounting of the polarimeter on the telescope.

A complete polarimetric exposure consists of a sequence of 4 subexposures, between which the retarder (for circular polarization Stokes $V$ ) or the instrument itself (for linear polarizations Stokes $Q$ and $U$ ) is rotated by $\pm 90^{\circ}$ (a procedure suggested by Semel et al. 1993). This has the effect of exchanging the beams within the whole instrument, and in particular switching the positions of the two orthogonally polarized spectra on the CCD. This observing procedure should in principle suppress all first-order spurious polarization signatures down to well below the noise level (typically 200-300 for our observations of $53 \mathrm{Cam}$ ).

Spectra obtained at the TBL using the MuSiCoS spectrograph and polarimeter were reduced using the ESPRIT reduction package, described by Donati et al. (1997). The ESPRITreduced spectra were then post-processed to improve the quality of the continuum normalization. The reduction procedure is described in some detail by Wade et al. (2000a).

The accuracy of the MuSiCoS circular and linear polarization measurements is supported by the results of Wade et al. (2000b). For a sample of magnetic Ap stars, Wade et al. have compared the net linear polarization, integrated across LeastSquares Deconvolved (LSD) mean Stokes $Q$ and $U$ profiles, with broadband linear polarization measurements published in the literature. Longitudinal magnetic field variations were also compared with published variations. The agreement in both cases is generally very good, and supports both the short-term and long-term accuracy of the polarimetric reference frame.

The log of observations of 53 Cam is shown in Table 1. Some observations (those obtained prior to Feb. 2000) have been described and analysed previously by Wade et al. (2000a,b) and by Bagnulo et al. (2001). A complete reduced spectrum of $53 \mathrm{Cam}$, in all four Stokes parameters, is reported by Wade (2002).

\subsection{Hydrogen Balmer lines}

In addition to the four Stokes parameter observations obtained at the Pic du Midi, we recorded a single unpolarized spectrum of 53 Cam with the MuSiCoS échelle spectrograph fed from the Cassegrain focus of the 2.5-m Isaac Newton Telescope (INT). The star was observed in January 2000 (rotational phase 0.647), using a $1024 \times 1024$ pixel CCD array. Complete coverage of the spectral region $3900-7000 \AA$ was obtained with a resolution of $\lambda / \Delta \lambda \approx 30000$ and a peak $S / N$ of 300 .

The spectrum was reduced using a standard set of the échelle reduction procedures in the IRAF package. Subsequent continuum normalization was performed with an IDL code, developed by Barklem et al. (2002). In their approach the difficulty of establishing accurate continuum in the échelle spectra is circumvented by detailed modelling of the $2 \mathrm{D}$ shape of the échelle blaze function, taking into account the configuration of an échelle spectrograph and using stellar exposures. Smooth 2D surfaces are iteratively fitted to the continuum in échelle orders with small number of absorption lines, while continuum in orders with high line density or deep absorption features is determined by interpolation. This method works very well even for lines that span several consecutive échelle orders (as it is the case for Balmer lines in the MuSiCoS spectrum of an A-type star).

The artificial flat-fielding procedure enabled us to extract and normalize hydrogen $\mathrm{H} \beta$ and $\mathrm{H} \gamma$ lines of $53 \mathrm{Cam}$ and to use these observations in constraining the stellar atmospheric parameters. Unfortunately, the $\mathrm{H} \alpha$ line from the MuSiCoS/INT spectrum could not be used due to a strong fringing at longer wavelengths, which could not be properly corrected.

\subsection{Spectrophotometry}

As an additional verification of the choice of model atmosphere parameters of 53 Cam we modelled its phase-averaged optical and near-UV flux distribution as tabulated by Pyper \& Adelman (1983). These authors obtained 14 spectrophotometric scans in the 3300-7850 A region using 20-30 A bandpasses. The shape of the 53 Cam flux distribution changes insignificantly with rotational phase. The scatter of individual scans around the mean is at the level of $0.01 \mathrm{mag}$, which is comparable to both the observational uncertainty and accuracy achievable by a theoretical fit.

According to the Hipparcos parallax $(10.16 \pm 0.77$ mas, ESA 1997), 53 Cam is located at about $100 \mathrm{pc}$ from the solar system at intermediate galactic latitude. In this direction the colour excess due to interstellar extinction is below 
Table 1. Spectropolarimetric measurements of 53 Cam used in the magnetic Doppler imaging inversions. Columns give the UT date of the observation, Stokes parameters observed (+ Stokes $I$ ), phases of mid-exposure for each observed Stokes parameter, the mean phase $\bar{\varphi}$ for all observed Stokes parameters, the maximum difference $\delta \varphi$ between the average and the phases of individual Stokes parameters, the heliocentric Julian Date, and the peak signal-to-noise ratio. Note that on $27 \mathrm{Feb}$. 2000, each of the $V, Q$ and $U$ observations was considered individually due to a larger-than-average $\delta \varphi$.

\begin{tabular}{ccccccccccc}
\hline \hline UT Date & \multicolumn{3}{c}{ Stokes } & \multicolumn{3}{c}{ Phases } & & & HJD & Peak \\
& \multicolumn{3}{c}{ Parameters } & $V$ & $Q$ & $U$ & $\bar{\varphi}$ & $\delta \varphi$ & $(-2400000)$ & $S / N$ \\
\hline 1997 Feb. 16 & $V$ & $Q$ & $U$ & 0.953 & 0.957 & 0.949 & 0.953 & 0.004 & 50496.4836 & 200 \\
1997 Feb. 17 & $V$ & & & 0.056 & & & 0.056 & & 50497.3150 & 140 \\
1997 Feb. 19 & $V$ & $Q$ & $U$ & 0.317 & 0.322 & 0.313 & 0.317 & 0.004 & 50499.4098 & 180 \\
1997 Feb. 21 & $V$ & $Q$ & $U$ & 0.565 & 0.569 & 0.560 & 0.565 & 0.005 & 50501.3948 & 150 \\
1997 Feb. 22 & $V$ & $Q$ & $U$ & 0.695 & 0.699 & 0.691 & 0.695 & 0.004 & 50502.4380 & 220 \\
1997 Feb. 23 & $V$ & $Q$ & $U$ & 0.815 & 0.819 & 0.811 & 0.815 & 0.004 & 50503.4034 & 100 \\
1997 Feb. 25 & $V$ & $Q$ & $U$ & 0.077 & 0.082 & 0.072 & 0.077 & 0.005 & 50505.5073 & 180 \\
1998 Feb. 04 & $V$ & $Q$ & $U$ & 0.923 & 0.927 & 0.919 & 0.923 & 0.004 & 50849.4237 & 330 \\
1998 Feb. 06 & $V$ & $Q$ & $U$ & 0.189 & 0.194 & 0.186 & 0.190 & 0.004 & 50851.5635 & 330 \\
1998 Feb. 08 & $V$ & & $U$ & 0.448 & 0.436 & & 0.442 & 0.006 & 50853.5891 & 230 \\
2000 Feb. 02 & $V$ & $Q$ & $U$ & 0.643 & 0.648 & 0.640 & 0.644 & 0.004 & 51577.6209 & 200 \\
2000 Feb. 09 & $V$ & $Q$ & $U$ & 0.515 & 0.520 & 0.511 & 0.515 & 0.004 & 51584.6172 & 160 \\
2000 Feb. 12 & $V$ & $Q$ & $U$ & 0.873 & 0.878 & 0.870 & 0.874 & 0.005 & 51587.4932 & 240 \\
2000 Feb. 24 & $V$ & $Q$ & $U$ & 0.371 & 0.375 & 0.367 & 0.371 & 0.004 & 51599.5130 & 190 \\
2000 Feb. 25 & $V$ & $Q$ & $U$ & 0.495 & 0.499 & 0.491 & 0.495 & 0.004 & 51600.5057 & 260 \\
2000 Feb. 27 & $V$ & & & 0.741 & & & 0.741 & & 51602.4825 & 220 \\
2000 Feb. 27 & & $Q$ & & 0.776 & & & 0.776 & & 51602.7658 & 220 \\
2000 Feb. 27 & & & $U$ & 0.750 & & & 0.750 & & 51602.5522 & 250 \\
2000 Mar. 03 & $V$ & $Q$ & $U$ & 0.359 & 0.363 & 0.366 & 0.363 & 0.004 & 51607.4729 & 140 \\
\hline
\end{tabular}

$0.2 \mathrm{mag} / \mathrm{kpc}$ (Lucke 1978) and has no appreciable effect on the overall shape of the flux distribution of $53 \mathrm{Cam}$, therefore we did not attempt to introduce a dereddening correction in the stellar spectrophotometric observations.

\section{Fundamental parameters of 53 Cam}

\subsection{Effective temperature and surface gravity}

In previous model atmosphere studies of 53 Cam authors typically found $T_{\text {eff }} \approx 8500 \mathrm{~K}$ and $\log g \approx 4.0$ (e.g., Landstreet 1988; Hubrig et al. 2000). We used these values of the effective temperature and surface gravity as the initial guess and attempted to verify model atmosphere parameters by a comprehensive analysis of all observational constraints available for $53 \mathrm{Cam}$. In addition to forming the basis for realistic spectrum synthesis calculations, accurate determination of the fundamental stellar characteristics enables us to derive the angle between the rotational axis and the line of sight (inclination angle $i$ ), required as an input for the magnetic Doppler modelling in Sect. 5.

Calculations of the model atmospheres and theoretical flux distributions were carried out using the ATLAS9 code of Kurucz (1993). We have chosen an opacity distribution function with 10 times solar metal abundance and a microturbulent velocity of $4 \mathrm{~km} \mathrm{~s}^{-1}$ to model line opacity in the atmosphere of $53 \mathrm{Cam}$. The high metal abundance is representative of the average atmospheric chemical composition of 53 Cam (Landstreet 1988). The enhanced microturbulence is used to mimic an overall increase in the line opacity due to magnetic intensification, which cannot be directly taken into account in standard ATLAS9 model atmospheres. In reality, the convective motions are expected to be suppressed by the very strong magnetic field of 53 Cam. Thus, apart from the choice of the opacity distribution function, we adopted zero micro and macroturbulent velocities in all spectrum synthesis calculations in this paper.

We used the average flux distribution of 53 Cam to constrain the stellar effective temperature. The slope of the Paschen continuum in the spectrophotometric observations of Pyper \& Adelman (1983) can be best matched with $T_{\mathrm{eff}}=$ $8400 \pm 150 \mathrm{~K}$. This value is fairly insensitive to the choice of the surface gravity and finds good agreement with the effective temperature of 53 Cam obtained in earlier studies. However, the observed Balmer jump requires an increase of the effective temperature to $T_{\text {eff }} \geq 9400 \mathrm{~K}$ or adopting an unrealistically large surface gravity of $\log g \approx 4.7$. The problem of fitting spectrophotometric observations of 53 Cam is illustrated in Fig. 1. We conclude that our approximate treatment of the anomalous line opacity in the model atmosphere and flux calculations for $53 \mathrm{Cam}$ is inadequate to reproduce some parts (such the Balmer jump) of the observed energy distribution, which are probably very much affected by the flux redistribution from the far ultraviolet and differential line-blanketing (Pyper \& Adelman 1983). In addition, the magnitude of the Balmer jump may be strongly affected by the presence of vertical gradients in chemical abundances (Wade et al. 2003). Thus, we decided to adopt the effective temperature inferred from the slope of the Paschen continuum and to estimate the spectroscopic surface gravity by fitting the hydrogen line profiles. 


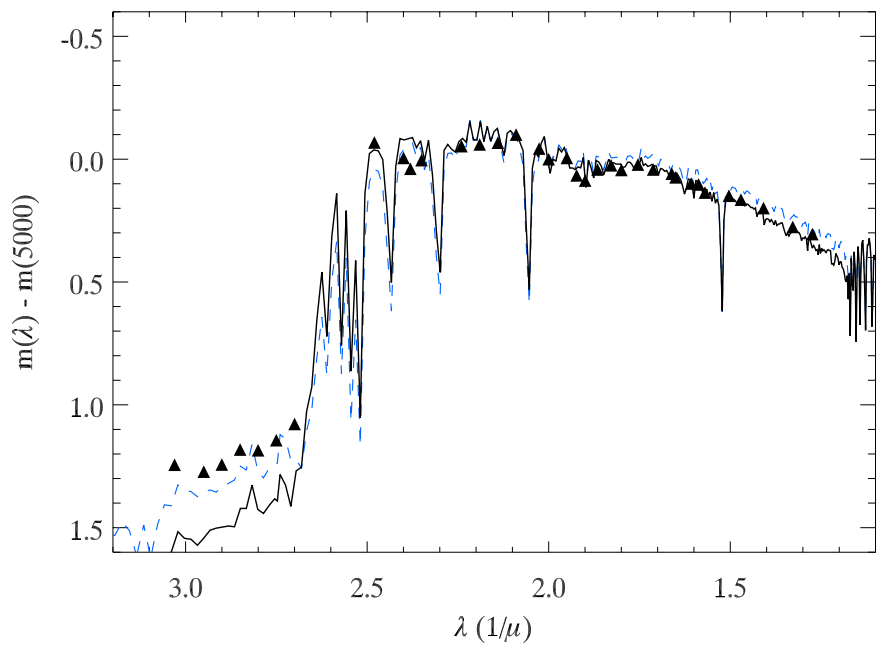

Fig. 1. The comparison between observed (triangles) and computed (lines) spectrophotometry of $53 \mathrm{Cam}$. The thick solid curve shows the energy distribution synthesized using the model atmosphere with $T_{\text {eff }}=8400 \mathrm{~K},[M / H]=+1.0$ and surface gravity $\log g=3.7$, as inferred from the profiles of the hydrogen Balmer lines. The dashed curve shows the synthetic energy distribution calculated with the stellar parameters providing the best overall fit to the observed spectrophotometry in the visual and near-UV spectral regions: $T_{\text {eff }}=$ $8500 \mathrm{~K},[M / H]=+1.0$ and $\log g=4.7$.

For the calculation of hydrogen lines we used a modified BALMER code of Peterson (1969). In our version of this program the Stark broadening tables of Vidal et al. (1973) were substituted by the more recent and presumably more accurate Stark broadening computations by Stehlé (1994). We found that the wings of the hydrogen $\mathrm{H} \beta$ and $\mathrm{H} \gamma$ lines of $53 \mathrm{Cam}$ can be fitted with $\log g=3.7 \pm 0.1$. The comparison between observed and calculated profiles of $\mathrm{H} \beta$ and $\mathrm{H} \gamma$ is presented in Fig. 2. This figure also illustrates that the high value of the surface gravity, suggested by the magnitude of the Balmer jump in spectrophotometric observations, is strongly discordant with the observed Balmer lines of 53 Cam.

\subsection{Luminosity and radius}

The accurate distance to $53 \mathrm{Cam}$, determined by the Hipparcos project, makes it possible to relate the stellar effective temperature and the basic physical parameters, such as luminosity and radius. Using the Hipparcos parallax (ESA 1997) and applying the Lutz-Kelker correction (Lutz \& Kelker 1973), Hubrig et al. (2000) found the absolute visual magnitude of 53 Cam, $M_{\mathrm{v}}=1.21$. A bolometric correction (Flower 1996), corresponding to the effective temperature of $53 \mathrm{Cam}$, is close to zero. Using the standard relations, connecting the effective temperature and bolometric magnitude with the stellar radius and luminosity,

$\log \left(L / L_{\odot}\right)=-\frac{M_{\mathrm{bol}}-M_{\mathrm{bol}}^{\odot}}{2.5}$

and

$R / R_{\odot}=\left(\frac{L}{L_{\odot}}\right)^{1 / 2}\left(\frac{T_{\mathrm{eff}}}{T_{\mathrm{eff}}^{\odot}}\right)^{-2}$, we found $L / L_{\odot}=24.9 \pm 1.2$ and $R / R_{\odot}=2.36 \pm 0.10$, where the solar bolometric magnitude was adopted to be $M_{\mathrm{bol}}^{\odot}=+4.71$ (Gray 1992).

\subsection{Inclination of the rotational axis}

The inclination $i$ of the stellar rotational axis can be constrained using its relation to the projected rotational velocity $v_{\mathrm{e}} \sin i$, stellar rotational period $P$ and radius $R$ :

$\sin i=\frac{P v_{\mathrm{e}} \sin i}{50.613} \frac{R_{\odot}}{R}$,

where the units for the rotational period and projected rotational velocity are taken to be days and $\mathrm{km} \mathrm{s}^{-1}$ respectively. Adopting the rotational period determined by Hill et al. (1998), $P=8.02681 \pm 0.00004$, and using $v_{\mathrm{e}} \sin i=12.5 \pm 0.5 \mathrm{~km} \mathrm{~s}^{-1}$, which gives the best fit to the observed rotational modulation of the 53 Cam spectra (see Sect. 5), we find $i=57^{\circ} \pm 5^{\circ}$ (or, equivalently, $i=123^{\circ} \pm 5^{\circ}$ ). This inclination angle agrees reasonably well with the values determined in the investigations of the magnetic geometry of 53 Cam by Landstreet $\left(1988, i=64^{\circ}\right.$ or $\left.116^{\circ}\right)$ and Bagnulo et al. $\left(2001, i=117^{\circ}\right)$.

\subsection{The position of the star in the $H-R$ diagram}

The stellar parameters determined in this section allow us to locate the position of $53 \mathrm{Cam}$ in the H-R diagram and estimate the stellar mass and age. Figure 3 allows us to interpret our values of the luminosity and effective temperature of 53 Cam using the theoretical evolutionary calculations of Schaller et al. (1992). This comparison yields a stellar mass $M=2.074 \pm 0.012 M_{\odot}$ and an age $\log ($ Age $)=8.789 \pm 0.038$, which corresponds to about $68 \%$ of the main sequence lifetime.

We can use the relation between the surface gravity and the stellar mass,

$\log g=\log g_{\odot}+\log \left(M / M_{\odot}\right)-2 \log \left(R / R_{\odot}\right)$,

to derive $\log g=4.010 \pm 0.046$. This value is marginally inconsistent with the spectroscopic surface gravity obtained from the hydrogen Balmer lines. About 0.1 dex of this discrepancy can be attributed to the helium depletion (typical for magnetic Ap stars) from the line-forming region in the atmosphere of 53 Cam. Unfortunately, the star is too cool to allow a meaningful helium abundance to be directly established from the optical or near-infrared He I absorption lines.

\subsection{Binarity}

53 Cam is known to be a single-lined spectroscopic binary with an orbital period of $P_{\text {orb }}=6.63$ years. In addition to the measurements of long term radial velocity variation, the binary system has been resolved with the speckle interferometric technique (McAlister et al. 1983). In principle, this allows one to attempt joint analysis of the radial velocity and interferometric data and to determine masses of individual components even in the absence of any spectroscopic information about the secondary star. Such an analysis has been recently carried out by Carrier et al. (2002), who suggested that the mass ratio 

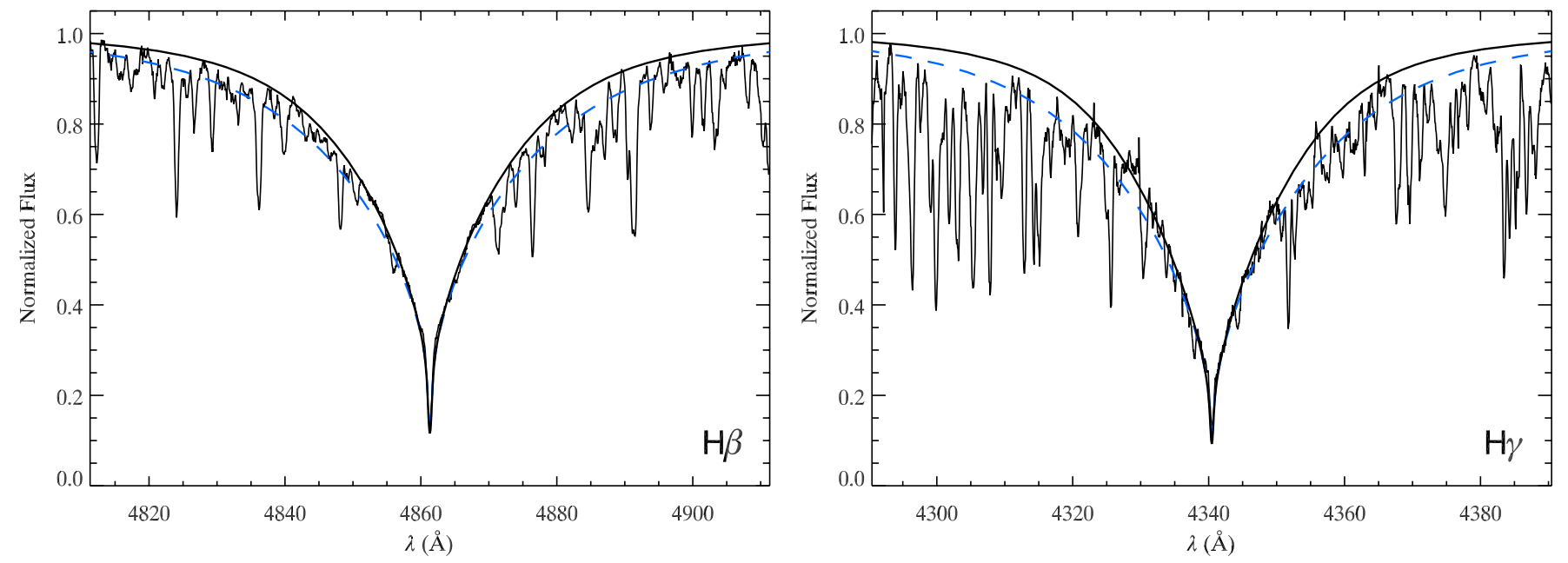

Fig. 2. The comparison between observed and computed hydrogen $\mathrm{H} \beta$ and $\mathrm{H} \gamma$ lines in the spectrum of 53 Cam. The thick solid lines correspond to the profiles calculated with $T_{\text {eff }}=8400 \mathrm{~K},[M / H]=+1.0$ and $\log g=3.7$. The dashed lines illustrate the effect of increasing the surface gravity to $\log g=4.5$.

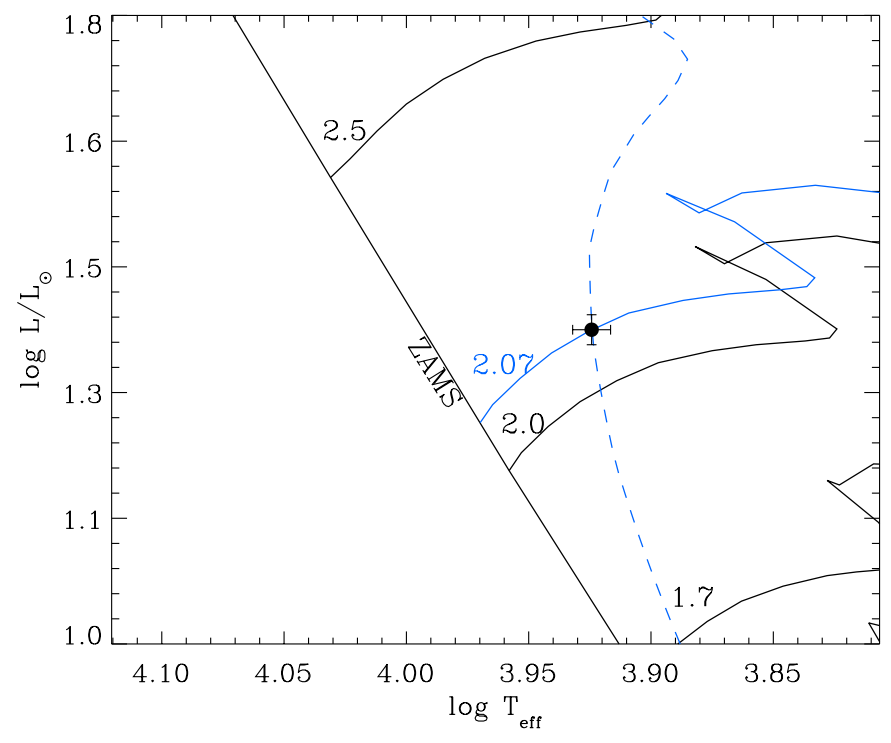

Fig. 3. The position of $53 \mathrm{Cam}$ in the H-R diagram is compared with the theoretical evolutionary tracks of Schaller et al. (1992), computed for solar metallicity and masses $M=1.7,2.0$ and $2.5 M_{\odot}$. The evolutionary track for the estimated mass of $53 \mathrm{Cam}, M=2.07 M_{\odot}$, was obtained by interpolating in the Schaller's et al. data. The dashed line shows the isochrone corresponding to the age of 53 Cam $(6.2 \times$ $10^{8}$ years).

$\left(M_{\mathrm{A}} / M_{\mathrm{B}}\right)$ of the components in 53 Cam system may be close to unity. If confirmed, such a conclusion would have important consequences for our study, since it implies that the contribution of the secondary star to the total spectrum could not be neglected in a modelling of magnetic geometry and abundance distribution of $53 \mathrm{Cam}$ (A). Therefore, in order to verify the results of Carrier et al. (2002), we modelled the same set of radial velocity and interferometric measurements using the code written by Tokovinin (1992). Our orbital elements are generally consistent with the parameters reported by Carrier et al. However, we found that some orbital parameters, in particular eccentricity, are strongly affected by the choice of relative
Table 2. Basic physical parameters of $53 \mathrm{Cam}$. The surface gravity listed in the table is a spectroscopic estimate obtained from the hydrogen Balmer lines.

\begin{tabular}{ll}
\hline \hline$T_{\text {eff }}(\mathrm{K})$ & $8400 \pm 150$ \\
$\log g$ & $3.70 \pm 0.10$ \\
$L / L_{\odot}$ & $24.9 \pm 1.15$ \\
$R / R_{\odot}$ & $2.36 \pm 0.10$ \\
$M / M_{\odot}$ & $2.074 \pm 0.012$ \\
$\log ($ Age $)$ & $8.789 \pm 0.038$ \\
\hline
\end{tabular}

weights given to the radial velocity and interferometric measurements (the errors of the former being uncertain due to the intrinsic rotational variability of the primary star, while the errors of the latter are difficult to assess as they are rarely quoted in the original studies). As a result, a mass ratio consistent at the $1 \sigma$ level with the orbital solutions for different combinations of relative weights ranges from about unity to $M_{\mathrm{A}} / M_{\mathrm{B}}=1.8$. This corresponds to a very large range of possible luminosity ratios $\left(L_{\mathrm{A}} / L_{\mathrm{B}} \approx 1 \div 9\right)$ and a secondary's spectroscopic contribution from prominent to negligible. Thus, at this point we can only conclude that orbital solution does not provide useful constraints on the mass ratio. This is primarily due to the insufficient number of interferometric measurements and their poor distribution with the orbital phase.

We searched all spectra of 53 Cam available to us for the spectroscopic signatures of the secondary star. None were found, thus it seems rather unlikely that $M_{\mathrm{A}} / M_{\mathrm{B}} \approx 1$. The analysis of Hipparcos astrometric observations of 53 Cam (Martin \& Mignard 1998) also supports this conclusion. Hence, in this paper we will adhere to the high mass ratio scenario and assume that spectroscopic contribution of the secondary star can be neglected.

Table 2 summarizes the fundamental parameters of $53 \mathrm{Cam}$ derived in our study. 


\section{Strategies for reconstructing magnetic field geometries of Ap stars}

The evolution of methods applied to study magnetic fields in Ap stars was traditionally determined by three factors: available data, sophistication of models and available computing resources. Early measurements of line splitting and circular polarization (Babcock 1958, 1960) together with a very successful oblique rotator model (Stibbs 1950) created a seemingly consistent picture of a magnetic Ap star. In this model a low-order multipolar, nearly axisymmetric surface magnetic field appears to be frozen into a rigidly rotating star, with the field axis tilted relative to the rotational axis. The magnetic field induces variations of chemical composition over the stellar surface by influencing the process of diffusive separation of chemical species. The distribution of chemical abundances is thus also not axisymmetric with respect to the rotational axis and is expected to follow the magnetic field geometry. The oblique rotator model predicts periodic changes in the observed intensity of spectral lines and explains smooth modulation of line splitting and circular polarization as a simple consequence of changes in geometrical aspect over the course of stellar rotation.

Under a number of reasonable simplifying assumptions, the observed low-order moments of spectral line profiles measured in intensity and circular polarization (so-called magnetic observables) can be directly related to the disk averages of the global magnetic field (e.g., Mathys 1989). This correspondence facilitated simple modelling of the magnetic geometries of Ap stars by straightforward comparison of observed and computed phase curves of the magnetic observables and derivation of the parameters of multipolar magnetic topologies (e.g., Bagnulo et al. 1999). Although such modelling has established itself as a powerful tool for initial estimates of basic parameters of global magnetic field geometries, a number of important limitations of the method have been identified. Most importantly, the moment technique is fundamentally limited because it is based on modelling secondary quantities derived from stellar line profiles. The method gives no guarantee that resulting magnetic models would provide an adequate description of the actual stellar polarization spectra. Furthermore, severe blending in complex spectra of Ap stars often precludes accurate measurements of magnetic observables. The moment method also ignores the effects of chemical spots and therefore cannot be applied to stars with appreciable surface variations of chemical abundances. It has been also recognized that magnetic observables are poorly suited to constrain higher-order multipolar components and are often unable to distinguish substantially different magnetic models since the latter may give very similar phase curves of magnetic observables. Thus, more detailed studies of the Stokes spectra of magnetic stars are essential to complement and test the usefulness of field models derived via application of the moment technique.

A complex problem of simultaneous mapping of abundance distributions and reconstruction of the magnetic geometry was attacked by Landstreet in the studies of 53 Cam (Landstreet 1988) and Babcock's star (Landstreet et al. 1989). These investigations were based on the analysis of the rotational modulation of the Stokes $I$ spectra and modelling field moments. Khokhlova et al. (2000) extended the method to both intensity and circular polarization line profiles. Both approaches still employed multipolar parameterizations to describe magnetic field, but made use of all information contained in the variation of line profiles. However, the computational demands related to synthesizing the spectra of magnetic stars became prohibitively large even for studies of a few spectral lines. As a result, previous attempts of simultaneous analyses of magnetic and abundance structures had to either assume a very simple parameterization of abundance distributions, a priori symmetric with respect to the magnetic field axis (in Landstreet's modelling), or use simplified and probably unrealistic analytical representation of the local Stokes profiles (in investigations by Khokhlova and collaborators).

Recently studies of the stellar magnetic fields have reached a new phase with the introduction of high-resolution spectropolarimeters at a number of medium-sized telescopes and first systematic measurements of the linear polarization in individual spectral lines of Ap stars (Wade et al. 2000a). Impressive advances in the quality of available polarization spectra have been matched by rapid increase in computing power and improvements in numerical techniques, making it possible to synthesize disk integrated Stokes stellar spectra in reasonable amount of time (Wade et al. 2001). These circumstances suggest that a magnetic Doppler imaging reconstruction of the chemical and magnetic structures based on direct analysis of high-resolution line profiles in Stokes parameters is a very promising tool for studies of Ap stars. It allows us to circumvent many limitations of traditional modelling of magnetic observables. Most importantly, magnetic Doppler imaging generalized to the analysis of all four Stokes parameters does not require an a priori parameterization of the global magnetic field structure and thus appears to be the only method capable of addressing a fundamental question: what is the actual structure of magnetic fields in Ap and related stars?

\section{Magnetic field geometry and iron abundance distribution of $\mathbf{5 3}$ Cam}

\subsection{Magnetic Doppler imaging with INVERS10}

The concept of magnetic Doppler imaging modelling of highresolution spectropolarimetric observations was discussed in detail by Piskunov \& Kochukhov (2002, Paper I). In that paper we introduced the new magnetic Doppler imaging code INVERS10 and described essential numerical techniques, such as the solution of the polarized radiative transfer equation, the integration of the emergent Stokes profiles over the stellar surface and optimization procedure, and parallel execution of the code on multi-processor supercomputers. We also refer reader to Paper I for the definition of the stellar and observer coordinate systems as well as the angles, specifying magnetic geometry and orientation of the stellar rotational axis. In the subsequent study (Kochukhov \& Piskunov 2002, Paper II) we assessed the performance and intrinsic limitations of the magnetic DI code with the help of numerical experiments, which consisted of the reconstruction of abundance 
and vector magnetic maps from simulated observations in four Stokes parameters.

It was convincingly demonstrated in Papers I and II that unique and reliable recovery of a global stellar magnetic map is possible with high-quality four Stokes parameter spectropolarimetric observational data. We argued that the information content of the Stokes IQUV profiles allows us to carry out the magnetic inversion without introducing any a priori assumptions about the large-scale magnetic field structure. In this case the role of regularization reduces to ensuring a consistency between the number of free parameters (determined by our choice of the stellar surface grid resolution) and information contained in the available observational data. Regularization also plays an important role in maintaining numerical stability and a smooth convergence of the minimization procedure. According to the results of our detailed analysis of various regularization strategies (Paper I), the Tikhonov regularization functional that we use in INVERS10 is appropriate for mapping global magnetic structures, expected in the atmospheres of Ap stars. On the other hand, the Maximum Entropy regularization method is successfully used in Zeeman-Doppler imaging of the complex patchy magnetic fields of active late-type stars (e.g., Donati 1999; Hussain et al. 2000), but shows poor performance when applied to reconstruction of the more uniform magnetic topologies of Ap stars.

In contrast to the magnetic mapping with complete Stokes parameter datasets, DI based on the analysis of only circular polarization observations is intrinsically non-unique and, therefore, it is strongly affected by the choice of the regularization function. This necessitates imposing additional constraints on the possible solutions of the inverse problem. We implemented this external information in the form of multipolar regularization, which forces the code to search for a magnetic map close to a combination of a dipole and non-linear quadrupole (see Paper I for more details). This modified version of our code was applied to the modelling of the Stokes $I$ and $V$ spectra of $\alpha^{2} \mathrm{CVn}$ (Kochukhov et al. 2002, Paper III). In the present study of the magnetic geometry of 53 Cam we analyse observations in all four Stokes parameters and use the original version of INVERS10, without applying multipolar regularization. The latter numerical technique is clearly unjustified in the case of 53 Cam because of the complexity of the magnetic field in this star. In our analysis the field is regularized using only conventional Tikhonov regularization function, which acts by imposing a local correlation between magnetic vectors in the neighbouring elements of the stellar surface. This effectively reduces the spatial resolution to the lowest value consistent with the information content of the data.

Numerical tests of the magnetic DI (Paper II) demonstrated that four Stokes parameter observations make it possible to address the problem of magnetic and abundance mapping of even very slowly rotating stars. In these objects, inaccessible for the conventional abundance DI, the Zeeman effect plays a major role in separating wavelength contributions of different surface elements and in this way helps to resolve stellar surface structures. 53 Cam belongs to this category of slowly rotating, strongly magnetic Ap stars, as its $v_{\mathrm{e}} \sin i$ is less or of the order of the Zeeman splitting of spectral lines.
However, in spite of general dramatic improvements in our ability to study magnetic structures of Ap stars with the combination of MuSiCoS spectropolarimetric data and the INVERS10 code, some characteristics of the available Stokes IQUV spectra of 53 Cam depart significantly from the properties of a desirable Stokes vector dataset. First, the spectral resolution of $R \approx 35000$ is significantly lower than $R \sim 10^{5}$, which according to our numerical experiments (see Paper II) is more appropriate for the analysis of the Stokes spectra of slowly rotating Ap stars. Secondly, the amplitude of the disk-integrated Stokes $Q$ and $U$ line profiles of 53 Cam is very low and a conspicuous linear polarization signal can be detected in only a few strong magnetically-sensitive spectral lines at a limited number of rotational phases (see Fig. 4). Thus, the magnetic inversion has to be constrained essentially by the amplitude of the linear polarization signatures. This reduction of the potential information content of the Stokes $Q$ and $U$ spectra has to be taken into account in the interpretation of the magnetic DI results.

\subsection{Reconstruction of the magnetic field topology}

Magnetic mapping of 53 Cam was started from several trial inversions using the Stokes IQUV profiles of Fe II $4923.93 \AA$. This spectral line is free from significant blends, has relatively accurate atomic parameters and shows clear rotational modulation in all Stokes parameters. Due to its high mean Landé factor $(z=1.69)$ and large equivalent width, the Fe II $4923.93 \AA$ line has one of the strongest linear polarization signatures in the spectra of Ap stars (Wade et al. 2000a). We found that the best fit to the Stokes $I$ spectra of this line can be achieved with $v_{\mathrm{e}} \sin i=12.5 \pm 0.5 \mathrm{~km} \mathrm{~s}^{-1}$. This value of the projected rotational velocity agrees well with $v_{\mathrm{e}} \sin i=13.0 \pm 1.5 \mathrm{~km} \mathrm{~s}^{-1}$ determined by Landstreet (1988).

Other important parameters, required as an input for the magnetic DI, include $i$ and $\Theta$ specifying respectively the tilt and the azimuth angle of the stellar rotational axis. Numerical experiments (Paper II) showed that magnetic inversion is relatively insensitive to uncertainty in the inclination angle $i$ (errors of $\approx 10^{\circ}$ can be tolerated). Therefore, we adopt $i$ determined in Sect. 3 from the fundamental parameters, $v_{\mathrm{e}} \sin i$ and rotational period of $53 \mathrm{Cam}$. This estimate of the inclination angle does not allow us to distinguish between $i$ and $180^{\circ}-i$. Such a distinction as well as an estimate of the azimuth angle $\Theta$ are possible by employing linear polarization measurements. In Paper II we demonstrated that, in principle, given high $S / N$ observations of the Stokes $Q$ and $U$ spectra, one can determine $\Theta$ by optimizing the fit to the observed rotational modulation of the Stokes profiles. Unfortunately, our Stokes $Q$ and $U$ observations of 53 Cam do not permit such a refined analysis. As it turned out, inversions with different $\Theta$ angles and two possible values of the inclination achieve similar quality of the fit to the observed Stokes spectra of the Fe II $4923.93 \AA$. The corresponding magnetic maps also do not differ appreciably, indicating that the reconstruction is unique despite large uncertainty in $\Theta$. Thus, as we suggested above, the low $S / N$ of the existing Stokes $Q$ and $U$ observations of 53 Cam indeed reduces somewhat our ability to recover 
$\begin{array}{llllll}\text { Stokes I } & (2.23 \%) & \text { Stokes Q } & (0.66 \%) & \text { Stokes U } & (0.60 \%)\end{array} \quad$ Stokes V $\quad(1.76 \%)$

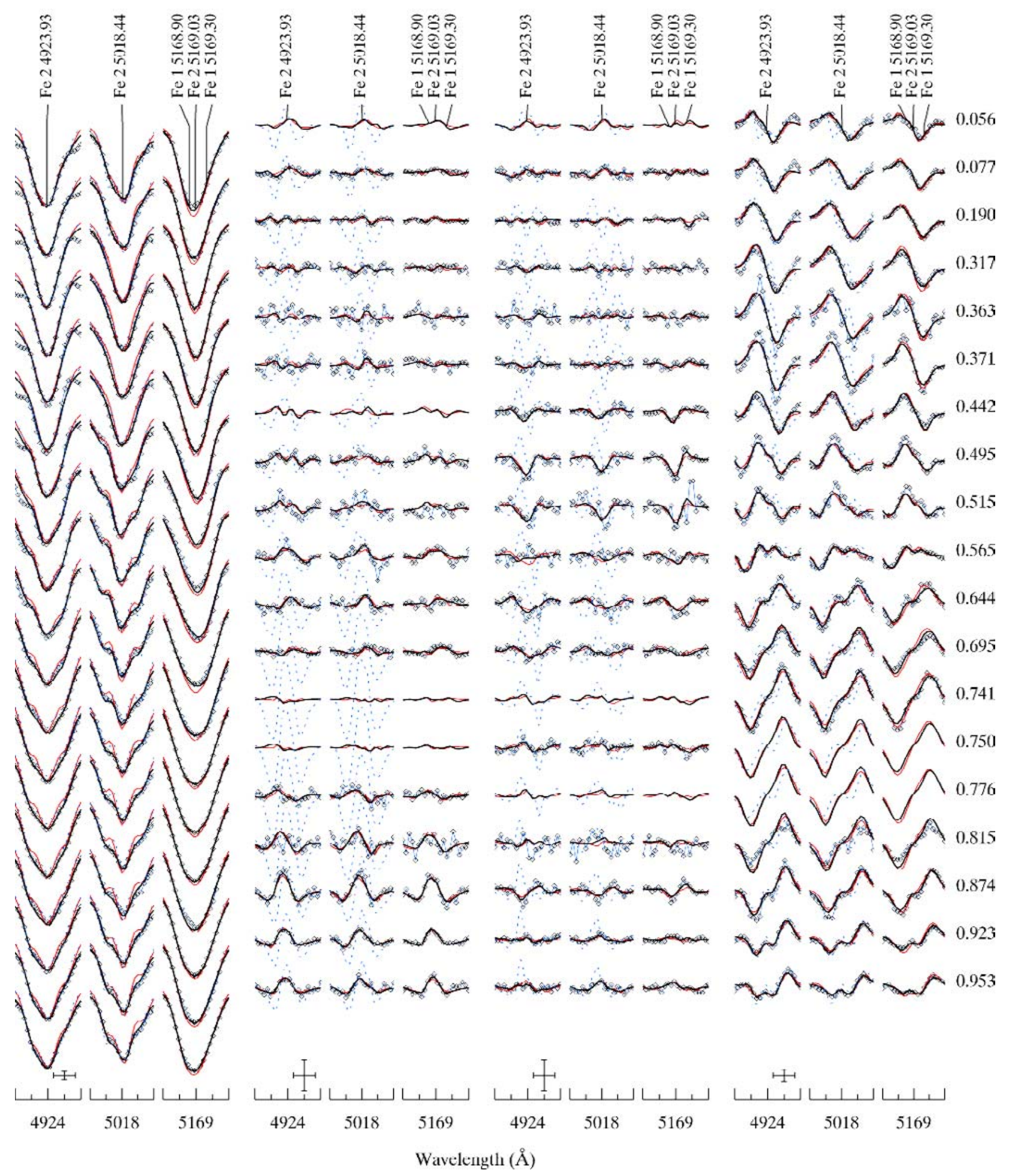

Fig. 4. The comparison between observed (symbols) and synthetic (solid and dotted curves) four Stokes parameters of the multiplet 42 Fe II lines in the spectra of $53 \mathrm{Cam}$. The thick curves show individual fits to the three Fe II lines obtained with the DI modelling of the magnetic field structure and iron abundance distribution. The thin profiles illustrate a similar fit achieved with no a priori assumption on the magnetic configuration, but adopting a constant Fe abundance. The dotted lines show the best-fit to the Stokes profiles of the Fe II 4923.93 and $5018.44 \AA$ assuming that the field is given by the superposition of a dipole and an arbitrarily oriented non-linear quadrupole. Spectra for the consecutive rotational phases are shifted in the vertical direction. Rotational phases are indicated in the column to the right of the Stokes $V$ panel. The bars at the lower left of each panel show the horizontal and vertical scale (1 $\AA$ and $5 \%$ of the Stokes $I$ continuum intensity respectively). The number in brackets at the top of each panel quotes mean deviation between observations and spectrum synthesis. 
orientation of the rotational axis solely on the basis of the high-resolution Stokes spectra. In this situation we have to adopt $i$ and $\Theta$ determined in previous studies of 53 Cam using much lower resolution, but higher $S / N$ broadband linear polarization (BBLP) observations. Remarkably, such measurements allowed Kemp \& Wolstencroft (1974) to constrain the inclination of the rotational axis of $53 \mathrm{Cam}, 90^{\circ}<i<180^{\circ}$ (i.e. an observer sees a clockwise stellar rotation), independently of the specific mechanism responsible for the polarization. In a recent quantitative analysis of the BBLP phase curves Bagnulo et al. (2001) deduced $i=117^{\circ}$ and $\Theta=139^{\circ}$, while in earlier study Landi Degl'Innocenti et al. (1981) obtained $\Theta=120^{\circ}$. Taking these results into account, we adopted $i=123^{\circ}$ and $\Theta=130^{\circ}$ for our modelling of the magnetic field structure of $53 \mathrm{Cam}$. We found that this orientation of the stellar rotational axis also leads to a marginally better fit to the Stokes profiles of the Fe II $4923.93 \AA$ line in comparison with the inversions assuming substantially different values of $\Theta$.

To verify the magnetic and abundance distributions derived from the Fe II $4923.93 \AA$, we repeated magnetic DI inversions using the four Stokes parameter spectra in the vicinity of the Fe II 5018.44 $\AA$ and Fe II 5169.03 $\AA$ lines. The latter spectral feature is blended by the Fe I lines at $\lambda 5168.90$ and $5169.30 \AA$, which were also taken into account in our spectrum synthesis. Atomic parameters of the Fe I and Fe II transitions were extracted from the VALD database (Kupka et al. 1999) and were checked by comparing synthetic spectra with the solar flux atlas (Kurucz et al. 1984). We found that $\log g f=-0.786$ given in the GFIRON list for the Fe I 5169.30 $\AA$ line is too high and has to be decreased to at least $\log g f=-2.15$ in order to match the solar spectrum.

Each of the three ionized iron lines was analysed independently, using homogeneous abundance and zero magnetic field as the initial guess. The magnetic topology and iron abundance distribution were recovered simultaneously in a self-consistent manner. The comparison between the four Stokes parameter observations of $53 \mathrm{Cam}$ and the synthetic profiles corresponding to the final individual best-fit magnetic topologies and abundance maps is presented in Fig. 4. Evidently, the fit to the intensity, linear and circular polarization spectra is very good. In particular, with the magnetic DI technique we are able to reproduce details of the Stokes $V$ profiles and at the same time obtain reasonable agreement between rotational modulation of the synthetic and observed Stokes $Q$ and $U$ parameters. This level of agreement between MuSiCoS observations and model predictions was not possible with any multipolar magnetic configurations obtained in previous studies of 53 Cam (see Bagnulo et al. 2001).

Figure 5 shows the spherical maps of the recovered orientation and strength of the magnetic field on the surface of $53 \mathrm{Cam}$. These images were reconstructed independently for each of the Fe II lines. Visual comparison of the maps suggests that the overall interagreement is satisfactory, although locally the discrepancy between the magnetic maps reaches $0.5-1.0 \mathrm{kG}$ in each of the vector magnetic components.
In comparison to the distributions of the field orientations, which are primarily constrained by the polarization spectra, the field strength maps are somewhat less coherent in the three independent inversions. This probably reflects the existence of marginal cross-talk between the field modulus and chemical abundance. When dealing with the spectra recorded at the resolution typical for the MuSiCoS data, the magnetic DI code may have difficulties to fully disentangle magnetic intensification from abundance spots, especially when modelling strongly saturated lines affected by inevitable errors in the continuum normalization and possible blending by unidentified spectral lines. It is likely that the latter problems are also responsible for the slightly different average iron abundance that we obtained from each of the Fe II lines $\left(\log \left(\mathrm{Fe} / N_{\text {tot }}\right)=-4.37,-4.14\right.$ and -4.61 for the Fe II 4923.93, 5018.44 and 5169.03 $\AA$ lines respectively).

An additional useful assessment of the agreement between the three magnetic maps emerges from analysis of the average magnetic topology. We found that the discrepancy between the Stokes $I Q U V$ line profiles predicted by such an average magnetic distribution and the four Stokes parameters corresponding to each of the three independent maps is indeed very small. This strongly supports the hypothesis that spectroscopic observations in four Stokes parameters provide a sufficient basis for a unique magnetic inversion, even in the case of the non-ideal Stokes IQUV dataset available for $53 \mathrm{Cam}$.

\subsection{Comparison with the magnetic observables}

As a supplementary test of the new magnetic model of 53 Cam, we computed the phase variation of the disk-integrated longitudinal magnetic field and field modulus. These model predictions were confronted with existing measurements of the magnetic observables. We stress that such a comparison is not defining in judging the reliability of the DI magnetic model because it has already passed the crucial test of reproducing the observed spectra in the four Stokes parameters.

In Fig. 7 we compare the model phase curve of $\left\langle B_{\mathrm{z}}\right\rangle$ with the photopolarimetric $\mathrm{H} \beta$ magnetic measurements by Borra \& Landstreet (1977) and Hill et al. (1998). The agreement between the synthetic and observed longitudinal field curves is good (except, perhaps, in small phase intervals around $\varphi=0.20$ and 0.75 ), given that these observations were not employed to constrain the magnetic model. The fit to the mean field modulus measurements by Huchra (1972) and Mathys et al. (1997) is shown in Fig. 8 and appears somewhat less satisfactory. Our magnetic model systematically predicts larger $\langle B\rangle$ between phases 0.1 and 0.5 by about $15 \%$. However, we recall that mean field modulus determinations using metal lines are rather uncertain for $53 \mathrm{Cam}$, owing to rotational Doppler effect and blending. This especially concerns the measurements by Mathys et al. (1997), who used only one diagnostic line of Fe II at $\lambda 6149.26 \AA$, which is strongly blended in the blue wing and, for the very strong field of $53 \mathrm{Cam}$, exhibits partial Paschen-Back rather than normal Zeeman splitting. 


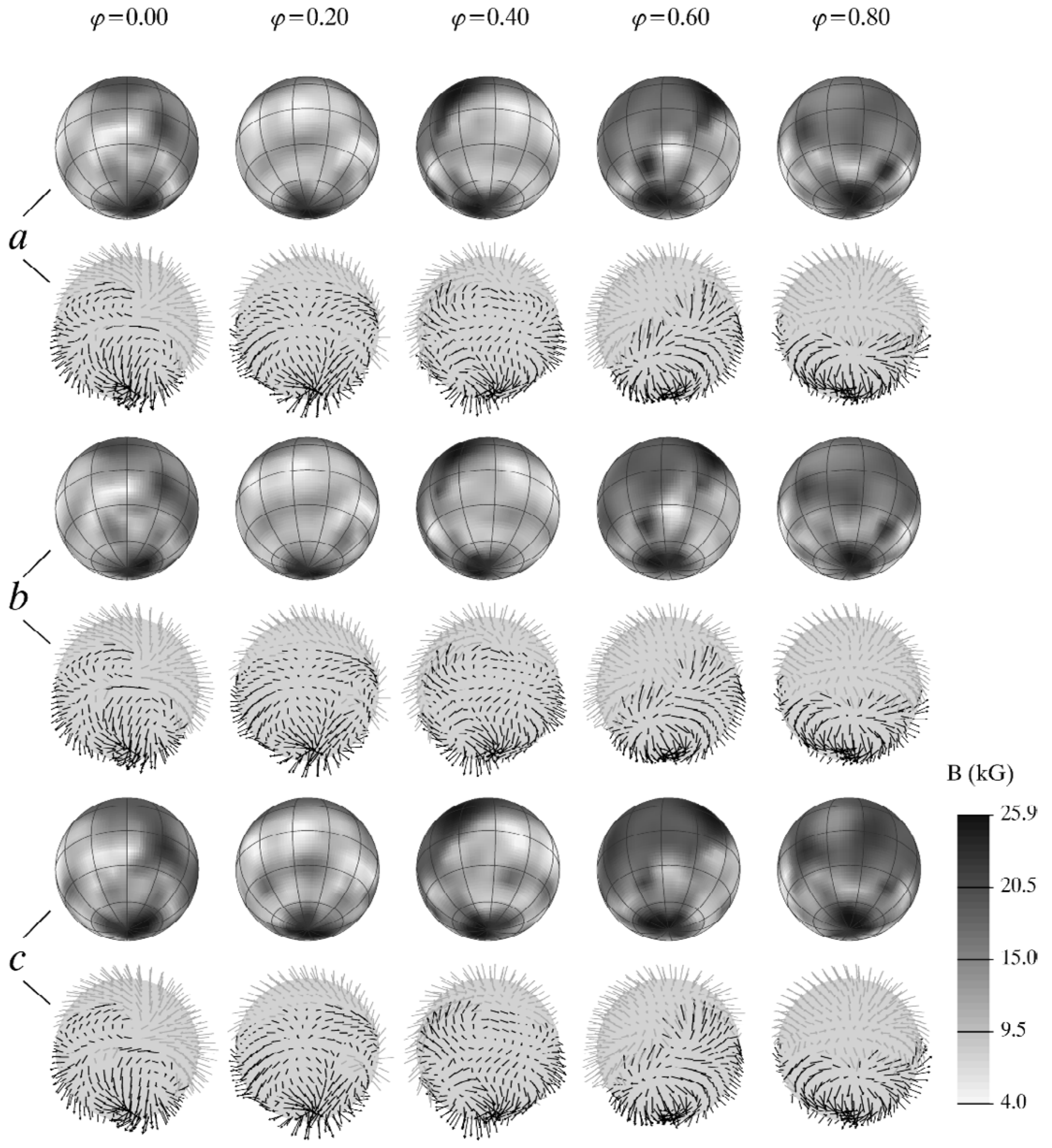

Fig. 5. Surface magnetic field distributions of 53 Cam derived from the Stokes $I Q U V$ profiles of the three Fe II lines. The star is shown at five equidistant rotational phases as indicated at the top of the figure. The aspect corresponds to the inclination angle $i=123^{\circ}$ and vertically oriented rotational axis. Magnetic Doppler imaging maps are presented separately for each of the Fe II lines modelled with INVERS10: a) $\lambda$ 4923.93 A, b) $\lambda 5018.44 \AA$ and c) $\lambda 5169.03 \AA$. In each pair of rows the greyscale plot (the upper panel) visualizes the distribution of field strength, while the lower panel shows the orientation of the magnetic vectors. In these vector maps the black arrows show field vectors pointing outside the stellar surface and the grey arrows correspond to the vectors pointing inwards. The arrow length is proportional to the field strength. 


\section{$a$}

$$
\varphi=0.00 \quad \varphi=0.20 \quad \varphi=0.40 \quad \varphi=0.60 \quad \varphi=0.80
$$
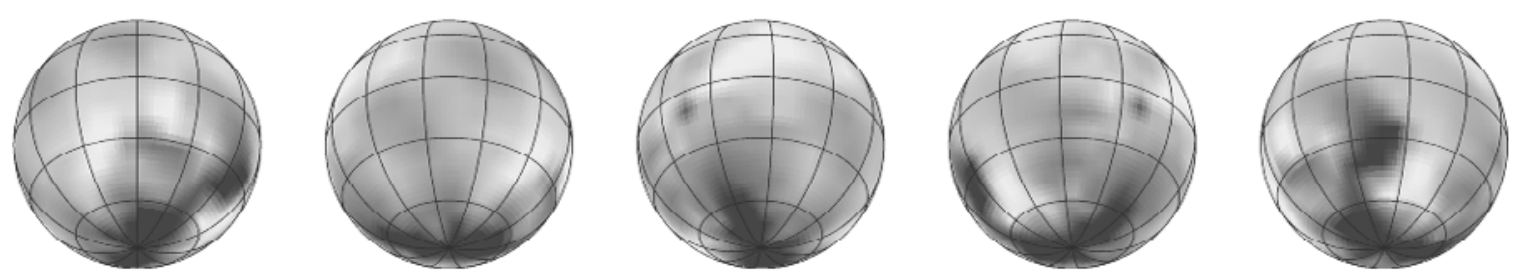

b
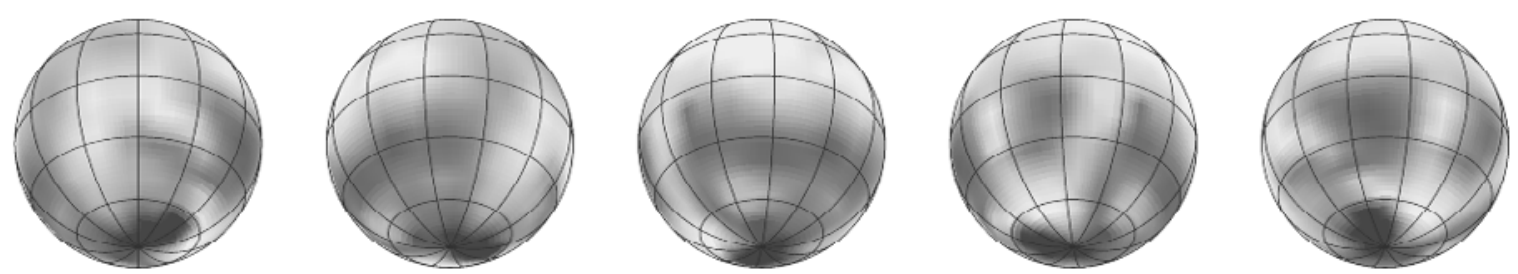

C
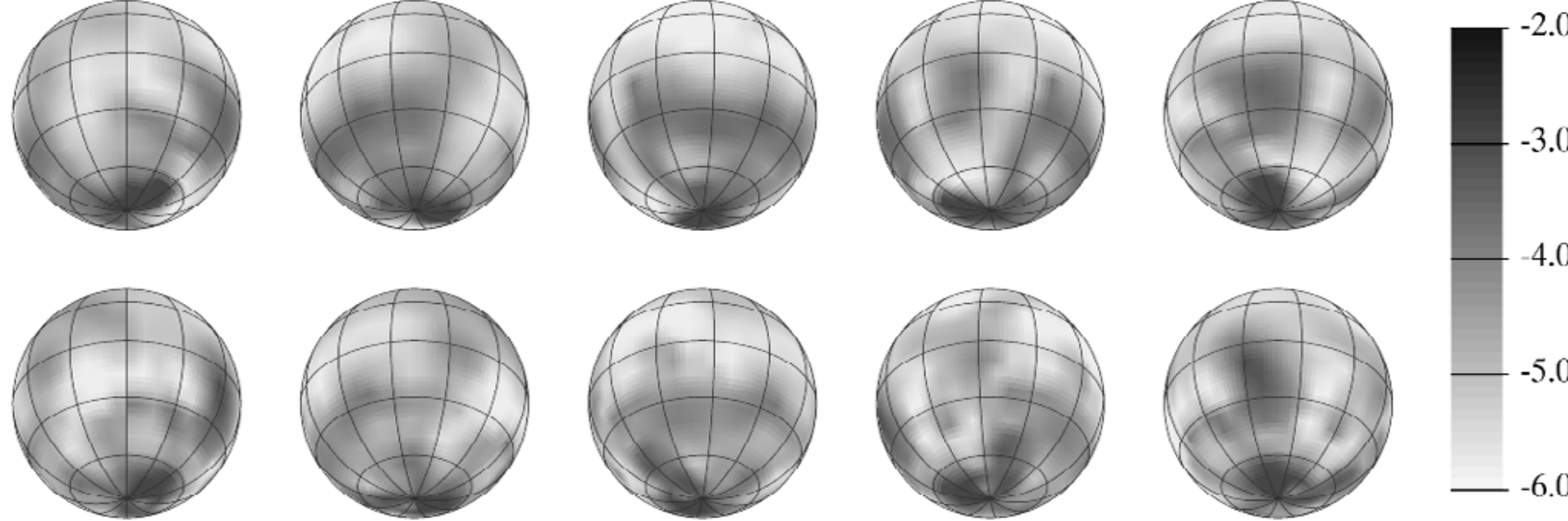

Fig. 6. Surface iron abundance distributions derived for 53 Cam using the Fe II lines: a) $\lambda 4923.93 \AA$, b) $\lambda 5018.44 \AA$ and c) $\lambda 5169.03 \AA$.

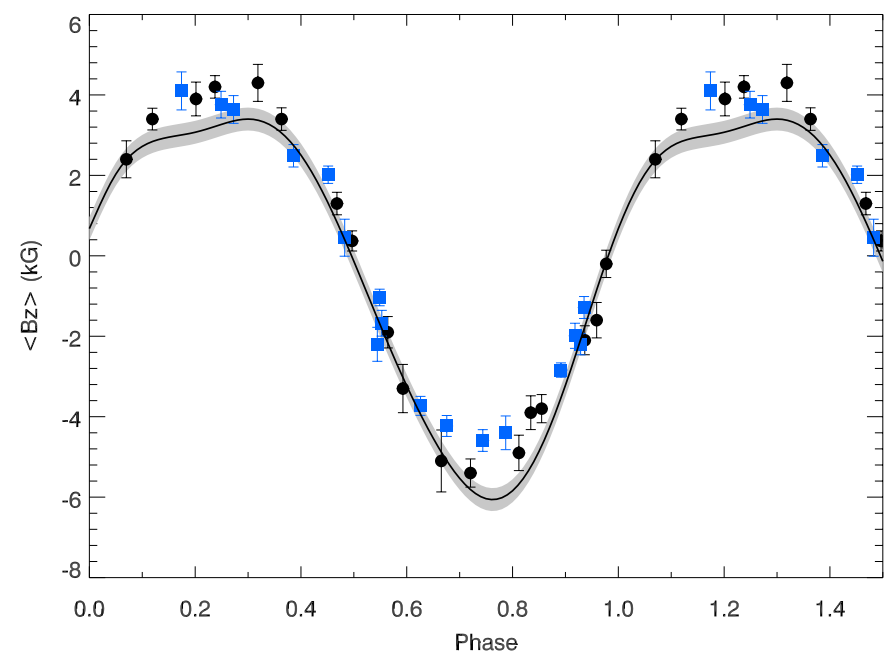

Fig. 7. The comparison between photopolarimetric $\mathrm{H} \beta$ measurements of the longitudinal field of 53 Cam (circles: Borra \& Landstreet 1977, squares: Hill et al. 1998) and variation of the longitudinal field predicted by the average magnetic Doppler map (solid line). The shaded curve corresponds to \pm one standard deviation of the longitudinal field curves, determined based on each of the three independent magnetic maps derived from the Fe II lines.

\subsection{Iron abudance distribution}

The maps of iron abundance derived simultaneously with the magnetic field geometries are shown in Fig. 6. Iron distributions recovered with INVERS10 are fairly complex and do not show any obvious correlation with the magnetic field geometry. The abundance maps suggest that the local Fe II concentration varies by about two orders of magnitude over the

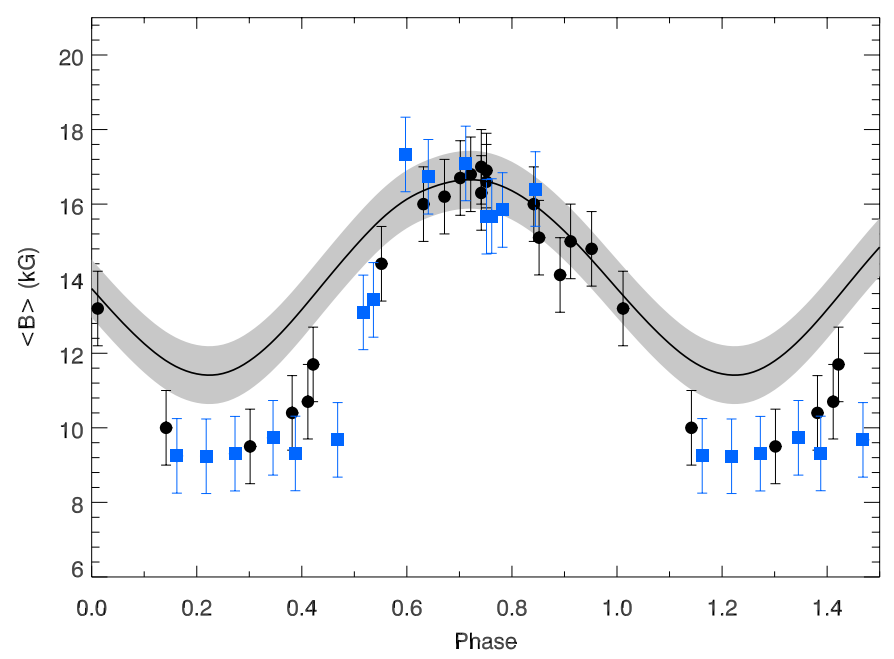

Fig. 8. The same as Fig. 7 for the comparison between mean field modulus measurements of 53 Cam (circles: Huchra 1972, squares: Mathys et al. 1997) and variation of the mean field strength predicted by the average magnetic Doppler map (solid line).

surface of 53 Cam. These significant horizontal iron abundance inhomogeneities are surprising because the Fe II lines do not show any spectacular profile variability that can be attributed to abundance spots. In addition, in earlier studies Landstreet (1988) and Bagnulo et al. (2001) showed that an inhomogeneous iron abundance was not required to fit the rotational modulation of the intensity profiles of Fe lines. Figure 4 shows that, if we adopt a uniform iron abundance, the fit to the Stokes $I$ profiles of the Fe II 4923.93 and $5018.44 \AA$ becomes noticeably worse. Thus, we tentatively suggest that iron abundance 
variations over the surface of 53 Cam are real. However, this conclusion certainly requires confirmation with the modelling of higher resolution and better $S / N$ spectropolarimetric observations. Moreover, since our choice of spectral lines used in magnetic inversions was necessarily restricted to strong saturated features (those showing conspicuous linear polarization signatures), the reconstructed iron abundance maps are very sensitive to small errors in continuum placement. This problem may be partially responsible for the poor concordance of the absolute iron abundances and the shapes of individual surface features seen in independent iron abundance maps.

\subsection{Multipolar analysis of the Stokes spectra and possible influence of abundance stratification}

Both magnetic and abundance DI maps of 53 Cam show a fairly complex structure. One may suspect that this is an artifact of the inversion technique, that may tend to interpret photon noise in terms of real features. To check this, we have tried to invert our set of Stokes profiles assuming that the field is given by the superposition of a dipole and a quadrupole arbitrarily oriented, and assuming a homogeneous abundance distribution. To perform this experiment we have made use of an older inversion code that was discribed by Bagnulo \& Wade (2001). Here we just recall that eleven free parameters are recovered after a $\chi^{2}$ minimization obtained through a Marquardt algorithm: the tilt and the azimuth angle of the rotation axis, the angle between the dipole axis and the rotation axis, the zero point phase, four angles specifying the orientation of the quadrupole, the dipole and quadrupole strength, and the equatorial velocity. (A more detailed definition of this framework is given by Landolfi et al. 1998.) The inversion code can model an arbitrary number of spectral lines, that are synthesized under the Milne-Eddington approximation. For 53 Cam we decided to invert simultaneously the Fe II lines at 4923.93 and $5018.44 \AA$ A. Several solutions were recovered, characterized by similar $\chi^{2}$ values. None of the corresponding sets of Stokes profiles could reproduce the observations in a satisfactory way. The best-fit to the observations obtained under the assumption of a dipole plus quadrupole magnetic configuration is compared to the model obtained with no a priori field parametrization in Fig. 4. Inspection of this figure shows that substantially better results can be obtained by allowing for an arbitrary magnetic configuration, which demonstrates the complexity of the magnetic field of 53 Cam and supports the reality of the results of the magnetic mapping with INVERs 10.

It should be noted that, in addition to surface chemical nonuniformities, vertical abundance stratification may also play an important role in shaping the observed Stokes spectra of Ap stars. It has been demonstrated that in such cool magnetic stars as $\beta \mathrm{CrB}$ (Bagnulo et al. 2001) and $\gamma$ Equ (Ryabchikova et al. 2002) the vertical chemical inhomogeneities are responsible for dramatic alternation of the relative strengths as well as the shapes of line profiles of many chemical elements. Neglecting strong abundance gradients in the magnetic and abundance Doppler imaging with INVERs10 can potentially produce spurious rotationally-symmetric surface structures, appearing due to a mismatch between real and synthetic local Stokes profiles. For instance, a steep increase of the concentration of chemical elements in deeper atmospheric layers gives rise to extended wings of strong spectral lines and wider line profiles in disk-integrated rotationally-broadened stellar spectra compared to a non-stratified case. This line profile anomaly may be misinterpreted as a low-latitude ring of enhanced elemental abundance by a Doppler imaging code like INVERs 10 , which assumes a constant chemical composition with depth. However, no such spurious latitudinal variation of the magnetic field strength or abundance is evident in our Doppler images reconstructed using the Fe II lines. Thus, it appears unlikely that possible iron abundance stratification has an appreciable influence on the three iron lines employed in the magnetic inversions. Therefore, vertical abundance gradients do not undermine our analysis of the magnetic field geometry of 53 Cam. This assumption is indirectly supported by the study of Bagnulo et al. (2001), who noted that stratification of ironpeak elements in the atmosphere of $53 \mathrm{Cam}$, though probably present, is not so prominent as compared to $\beta \mathrm{CrB}$.

\section{Abundance distributions of $\mathrm{Si}, \mathrm{Ca}, \mathrm{Ti}$ and $\mathrm{Nd}$}

After deriving the magnetic field topology of 53 Cam from the four Stokes parameter spectra, we can use INVERs10 to obtain surface distributions of other chemical elements. As we explained above, the resolution of the MuSiCoS spectra is barely sufficient to explore the fine details of the geometry of abundance spots on the surface of 53 Cam. Ideally, we would like to employ very high resolution Stokes $I$ observations for the abundance mapping. Nevertheless, in this section we will try to use MuSiCoS observations to derive preliminary abundance maps for a few chemical elements showing the most dramatic spectrum variability. In these abundance inversions we use the average magnetic map derived from the Fe II lines and simultaneously fit the Stokes $I$ and $V$ spectra. For 53 Cam these Stokes parameters have comparable ratios of the full amplitude to the noise level and we can expect that the Stokes $V$ spectra are sufficiently influenced by inhomogeneous abundances (see Paper III) to complement the information content of the Stokes $I$ profiles and aid in the abundance mapping.

In this exploratory study we have chosen to reconstruct surface abundance distributions of $\mathrm{Si}, \mathrm{Ca}$, Ti and $\mathrm{Nd}$. For each of the chemical elements, except $\mathrm{Nd}$, inversions were carried out using two spectral lines. The final fit to the Stokes $I$ and $V$ spectra of the seven lines is presented in Fig. 9, while Fig. 10 shows spherical maps of the corresponding abundance distributions and compares them with the average magnetic map of 53 Cam.

The map of Si was recovered from the two strong red lines of Si II at $\lambda 6347.11$ and $6371.37 \AA$. The agreement between the observed and computed intensity and circular polarization profiles is reasonably good. A significant part of the variability of the Si II lines, in particular the doubling of Si II $6371.37 \AA$, is entirely due to magnetic field. The silicon abundance is dominated by a strong rotationally symmetric component, which suggests some influence of the vertical stratification of Si.

The surface distribution of $\mathrm{Ca}$ was obtained using the Ca I 6162.17 and $6439.07 \AA$ lines. We found that the calcium 
Stokes I

$(1.78 \%)$

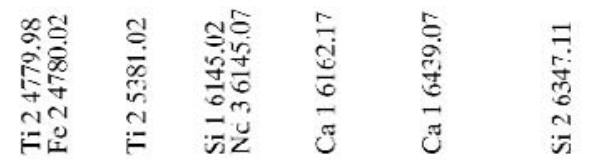

Le $f(p)$

b t $\mathrm{V}$

L

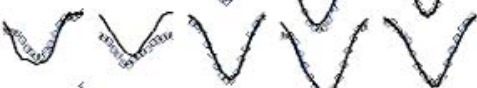

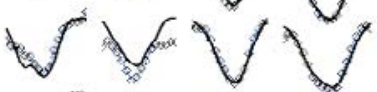

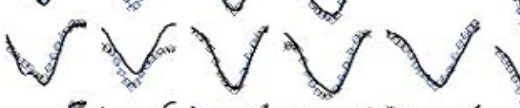

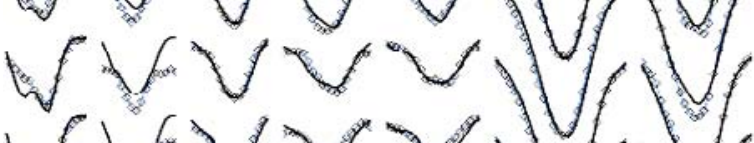

w v v w w

bif $v a v$ V

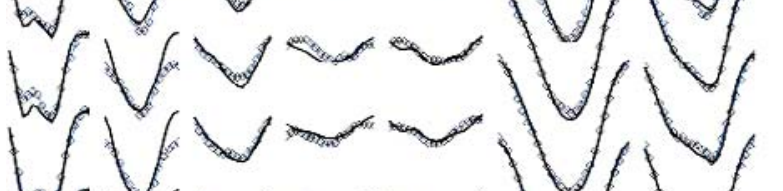

W
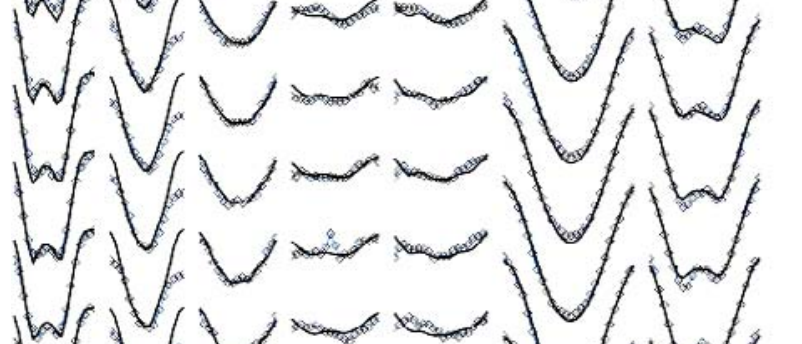

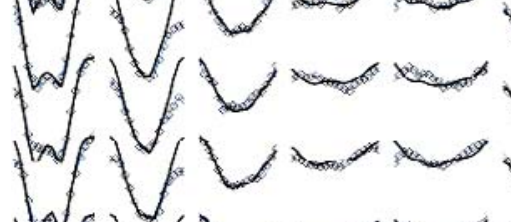

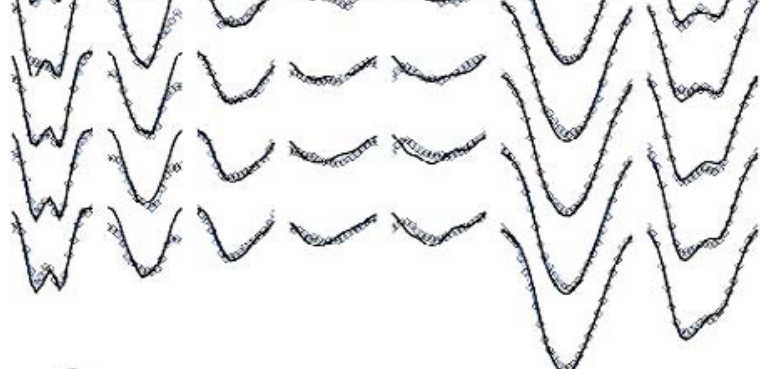

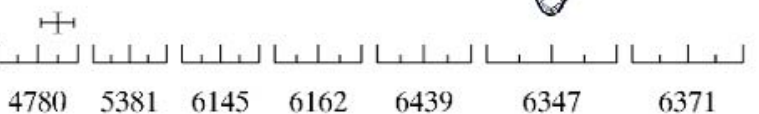

Stokes V

$(1.16 \%)$
0.056

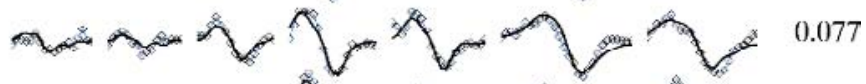

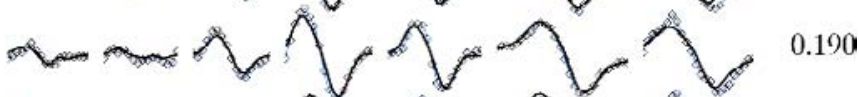

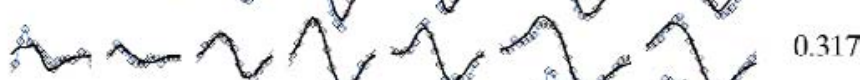

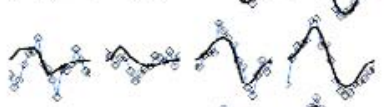

-

की

ㄴ.

0.363

0.371

r.

0.442

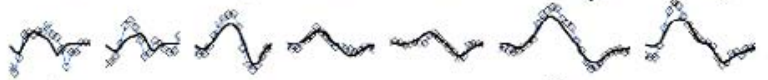

0.495

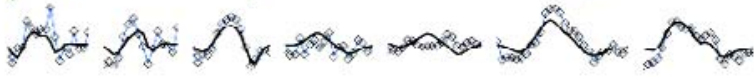

0.515

$\checkmark$ A

0.565

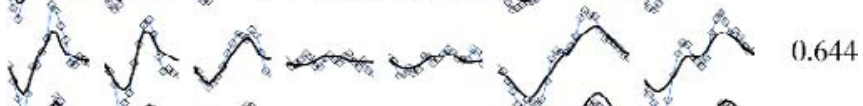

V

V

$\sqrt[v]{ } \wedge \sim \wedge^{0.750}$

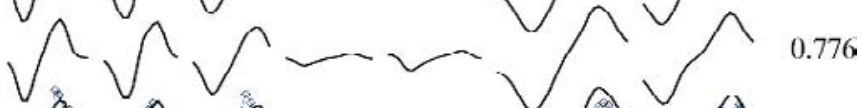

fo A

$\sqrt{*} v^{\prime} f^{0.874}$

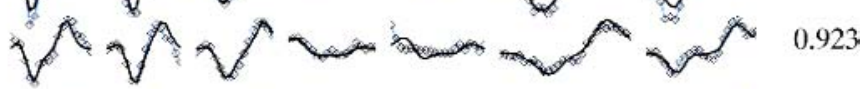

ฟ

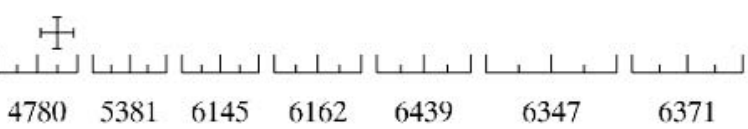

Wavelength (̊̊)

Fig. 9. The comparison between observed (symbols) and synthetic (solid curves) Stokes $I$ and $V$ parameters of the Si II, Ca I, Ti II and Nd III lines used in reconstruction of the element abundance distributions. The format of the figure is similar to that of Fig. 4.

map features an area of overabundance, associated with weak field strength and positive line-of-sight magnetic orientation. This relation between the calcium abundance map and magnetic topology, as well as the range of $\mathrm{Ca}$ abundance variation, are in perfect agreement with Landstreet's (1988) results.
Two lines of singly ionized Ti, $\lambda 4779.98$ and $5381.02 \AA$, were used to reconstruct the distribution of this element. The titanium map is characterized by a large spot visible between phases 0.5 and 0.0. This area of $\mathrm{Ti}$ overabundance is more extended than the $\mathrm{Ca}$ spot and corresponds to the stronger 


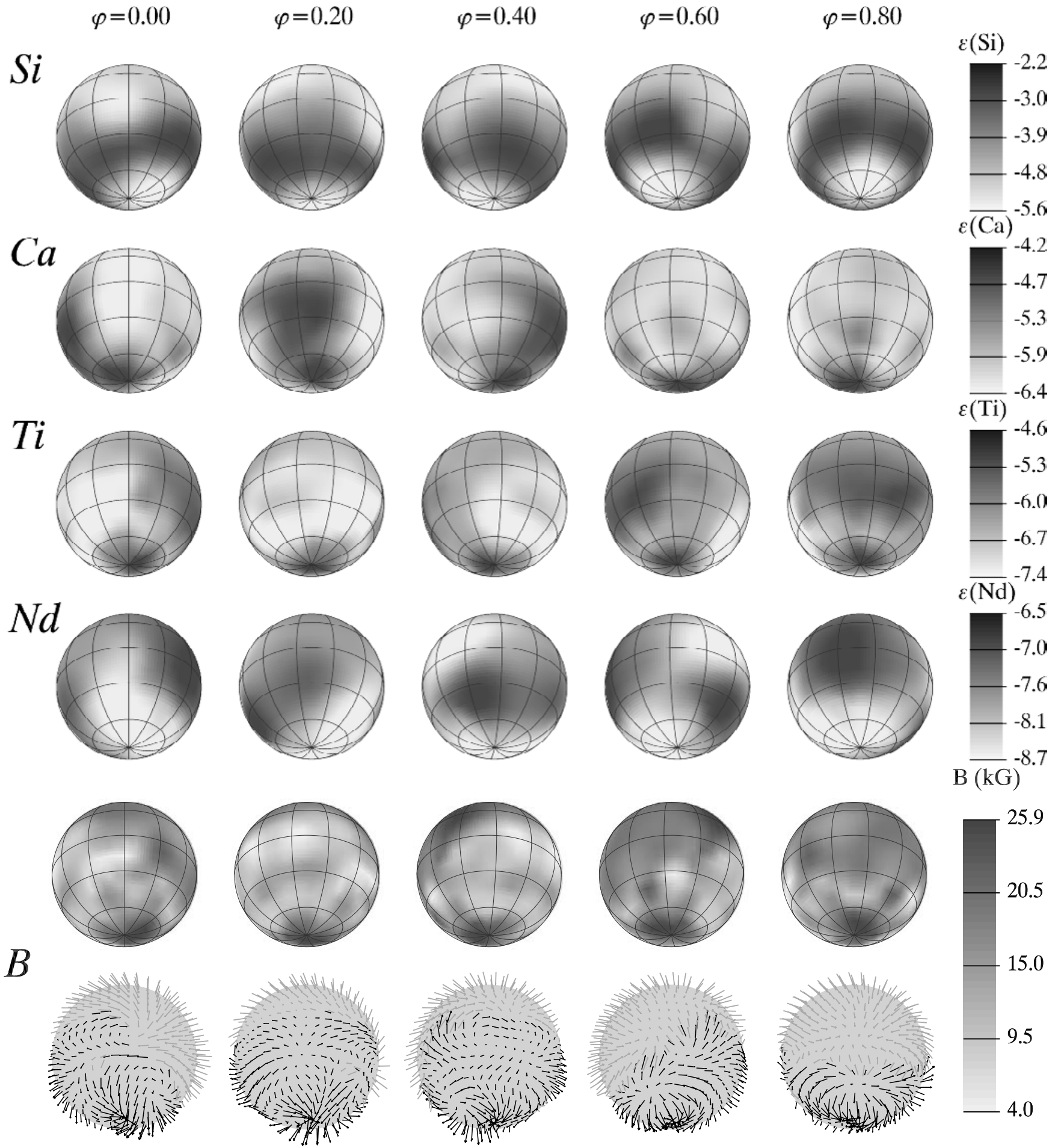

Fig. 10. Surface abundance distributions of $\mathrm{Si}, \mathrm{Ca}$, Ti and $\mathrm{Nd}$ reconstructed from the Stokes $I$ and $V$ line profiles of $53 \mathrm{Cam}$. The two lower panels show the average magnetic map reconstructed from the Fe II lines.

negative part of the magnetic geometry. This again agrees well with the outcome of Landstreet's (1988) investigation, although his $\mathrm{Ti}$ abundance is on average lower by $\approx 0.5 \mathrm{dex}$ compared to the Ti map derived in our study.

The surface distribution of $\mathrm{Nd}$ was reconstructed using the doubly ionized line at $\lambda 6145.07 \AA$. Its astrophysical oscillator strength was taken from the study of Dolk et al. (2002) and the $\log g f$ of the blending Si I $6145.02 \AA$ line was adjusted by fitting the solar spectrum. We found that the variation of the $\mathrm{Nd}$ III concentration over the surface of 53 Cam reaches 2 dex and the distribution of this element is dominated by the two spots visible around phases 0.5 and 0.9 . 


\section{Discussion}

\subsection{Multipolar expansion of the magnetic DI map}

Visual inspection of the magnetic Doppler imaging maps of 53 Cam indicates that, despite a fairly simple quasi-dipolar distribution of the radial magnetic component, the topology of the tangential field and especially the field strength appear to be very complex. Clearly, a more thorough quantitative assessment is required to fully characterize and understand the results of the magnetic inversions. Expansion into a spherical harmonic series provides a convenient mathematical apparatus for the analysis of vector fields specified on spherical surfaces. Multipolar analysis of the DI maps obtained for $53 \mathrm{Cam}$ was carried out using the multipolar expansion technique introduced in Paper I. In contrast to the regular integral approach of obtaining spherical harmonic coefficients, our method is formulated as a linear least-squares problem, with multipolar coefficients determined from a solution of a set of linear equations. This form of multipolar expansion has an important advantage in that it does not depend directly on the sampling of a vector field. Furthermore, different surface areas can be treated with different weights and the invisible part of a vector field can be excluded, which is especially useful in stellar applications.

The stellar magnetic field is represented as a superposition of the poloidal and toroidal vector fields:

$\boldsymbol{B}=-\sum_{\ell=1}^{N} \frac{4 \pi}{2 \ell+1} \sum_{m=-\ell}^{\ell}\left(c_{\ell m}^{(\mathrm{p})} \nabla^{(\mathrm{p})}+c_{\ell m}^{(\mathrm{t})} \nabla^{(\mathrm{t})}\right) \frac{\overline{\mathrm{Y}}_{\ell m}(\theta, \varphi)}{r^{\ell+1}}$,

where $\overline{\mathrm{Y}}_{\ell m}(\theta, \varphi)$ are real (tesseract) spherical harmonics, $c_{\ell m}^{(\mathrm{p})}$ and $c_{\ell m}^{(\mathrm{t})}$ are expansion coefficients and vector operators defining poloidal and toroidal components are given in spherical coordinates by

$\nabla^{(\mathrm{p})}=\left\{\frac{\partial}{\partial r}, \frac{\partial}{\partial \theta}, \frac{1}{\sin \theta} \frac{\partial}{\partial \varphi}\right\}$ and $\nabla^{(\mathrm{t})}=\left\{0, \frac{1}{\sin \theta} \frac{\partial}{\partial \varphi},-\frac{\partial}{\partial \theta}\right\}$.

Multipolar analysis was performed for $N=15$. This gives 550 poloidal and toroidal expansion coefficients and corresponds to the smallest angular scales roughly matching the average effective resolution $\left(\approx 10^{\circ}\right)$ of the stellar surface grid employed in our magnetic inversions. Contribution of the field components with different $\ell$ values were characterized by computing rms averages over $m$ values:

$C_{\ell}^{(\mathrm{p}, \mathrm{t})}=\sqrt{\sum_{m=-\ell}^{\ell} \frac{\left(c_{\ell m}^{(\mathrm{p}, \mathrm{t}}\right)^{2}}{2 \ell+1}}$.

Parameters $C_{\ell}^{(\mathrm{p})}$ and $C_{\ell}^{(\mathrm{t})}$ are plotted in Fig. 11 as a function of $\ell$ and the angular scale. This figure confirms the complexity of the magnetic distribution of 53 Cam inferred with the magnetic DI. Evidently, the low-order $(\ell=1-3)$ poloidal components are not dominant in the magnetic topology. Instead $C_{\ell}^{(\mathrm{p})}$ reach maximum at $\ell \approx 5-6$ (angular scales $30^{\circ}-40^{\circ}$ ) and then smoothly decreases to zero, with the strength of the high-order components $(\ell \approx 10)$ still comparable to the dipolar contribution. On the other hand, low-order terms are the most important among the toroidal expansion coefficients $C_{\ell}^{(\mathrm{t})}$. In the range of

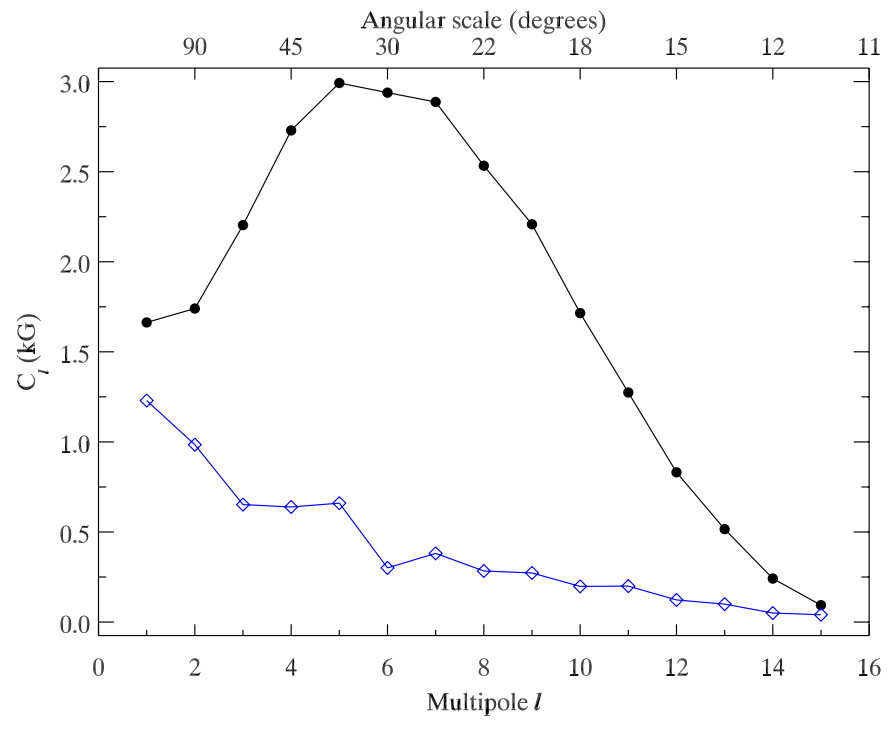

Fig. 11. Results of multipolar expansion of the DI magnetic map obtained for $53 \mathrm{Cam}$. The average poloidal (filled symbols) and toroidal (open symbols) expansion coefficients are plotted as a function of degree $\ell$ and angular scale on the stellar surface.

angular scales between $\ell=1$ and 3 their amplitude is comparable to the strength of the corresponding poloidal terms.

We stress that the high-order multipolar model examined in this section is meant to be used only for a general analysis of the scales of magnetic structures in the DI map of $53 \mathrm{Cam}$. The multipolar model does not provide a perfect approximation of all fine details in the actual magnetic image recovered by INVERS10, since residual discrepancies between the multipolar model and Doppler image are still at the level of $1-3 \mathrm{kG}$. A better approximation cannot be obtained by including higher terms in the multipolar expansion, because this problem becomes poorly constrained and computationally unstable for $\ell \gtrsim 15$ and our surface grid containing 695 elements. We suggest that a much more reliable and accurate way of analysing multipolar magnetic structures in $53 \mathrm{Cam}$ and other Ap stars can be achieved by optimizing expansion coefficients entering Eq. (5) through the direct comparison between observed and computed four Stokes parameter stellar spectra. A somewhat similar approach has been already explored by Hussain et al. (2001) in the context of investigation of weak magnetic fields in active late-type stars using circular polarization spectra. However, extension of this technique to all four Stokes parameters and imaging strong fields of Ap stars requires significant modifications of our inversion code and lies outside the scope of the present study.

\subsection{Consistency with Maxwell's equations}

The reconstructed vector magnetic field of 53 Cam must fulfil Maxwell's equations. An analysis of the field map in this context therefore serves as one check of the reliability of the maps. We will discuss in particular the behaviour of the reconstructed field with respect to the (local) equations:

$\nabla \cdot \boldsymbol{B}=0$ 
and

$\nabla \times \boldsymbol{B}=\mu_{0} \boldsymbol{J}+\mu_{0} \epsilon_{0} \frac{\partial \boldsymbol{E}}{\partial t}$,

as well as the (global) equation:

$\oint \boldsymbol{B} \cdot \mathrm{d} \boldsymbol{S}=0$.

In Eqs. (8), (9) and (10), $\boldsymbol{B}$ is the magnetic flux density, $\boldsymbol{J}$ is the current density, and $\boldsymbol{E}$ is the electric field. Under the assumption of a steady-state electric field $(\partial \boldsymbol{E} / \partial t=0)$, these equations, in spherical coordinates, reduce to:

$\frac{1}{r^{2}} \frac{\partial\left(r^{2} B_{r}\right)}{\partial r}+\frac{1}{r \sin \theta} \frac{\partial B_{\theta} \sin \theta}{\partial \theta}+\frac{1}{r \sin \theta} \frac{\partial B_{\phi}}{\partial \phi}=0$

and

$\frac{1}{r \sin \theta}\left(\frac{\partial B_{\phi} \sin \theta}{\partial \theta}-\frac{\partial B_{\theta}}{\partial \phi}\right)=\mu_{0} J_{r}$

$\frac{1}{r}\left(\frac{1}{\sin \theta} \frac{\partial B_{r}}{\partial \phi}-\frac{\partial r B_{\phi}}{\partial r}\right)=\mu_{0} J_{\theta}$

$\frac{1}{r}\left(\frac{\partial r B_{\theta}}{\partial r}-\frac{\partial B_{r}}{\partial \theta}\right)=\mu_{0} J_{\phi}$

and

$\oint B_{r} \mathrm{~d} S=0$.

Equations (11), (13) and (14) cannot be solved uniquely using our vector field map of $53 \mathrm{Cam}$, because the vertical gradients of the field components $\partial B_{x} / \partial r$ are not known. We point out that Eq. (11) can in principle be employed to constrain the vertical gradient of the radial field component $B_{r}$, assuming that the reconstructed field conforms to Maxwell's equations. Such an analysis is however outside the scope of this study.

Equation (12) allows for the determination of the vertical current density $J_{r}$ based on the reconstructed surface magnetic field. The current density provides a direct measure of the nonpotentiality of the surface magnetic field, and solar magnetic field studies reveal that large vertical current densities are associated with some activity phenomena (e.g. Wang 1993). Large inferred current densities in the atmosphere of an Ap star would perhaps be worrisome, as activity phenomena (e.g. flares) are not observed in these objects.

When Eq. (12) is applied to our magnetic map of 53 Cam, we obtain a map of the surface vertical current density, which is shown in Fig. 12. The maximum current densities, of several $\mathrm{mA} \mathrm{m}{ }^{-2}$, are around 5-10 times smaller than those observed in solar active regions (e.g. Metcalf et al. 1994). However, Abramenko et al. (1990) suggest that spots in active regions begin to appear at a threshold value of about $1 \mathrm{~mA} \mathrm{~m}^{-2}$. On the other hand, active regions on the sun cover surface regions which are physically much smaller than the smallest resolved element in our map $\left(\sim 10^{\circ}\right)$. It is therefore unclear whether current densities with amplitudes such as those we infer in the atmosphere of $53 \mathrm{Cam}$ are consistent or not with the observed behaviour of Ap stars.

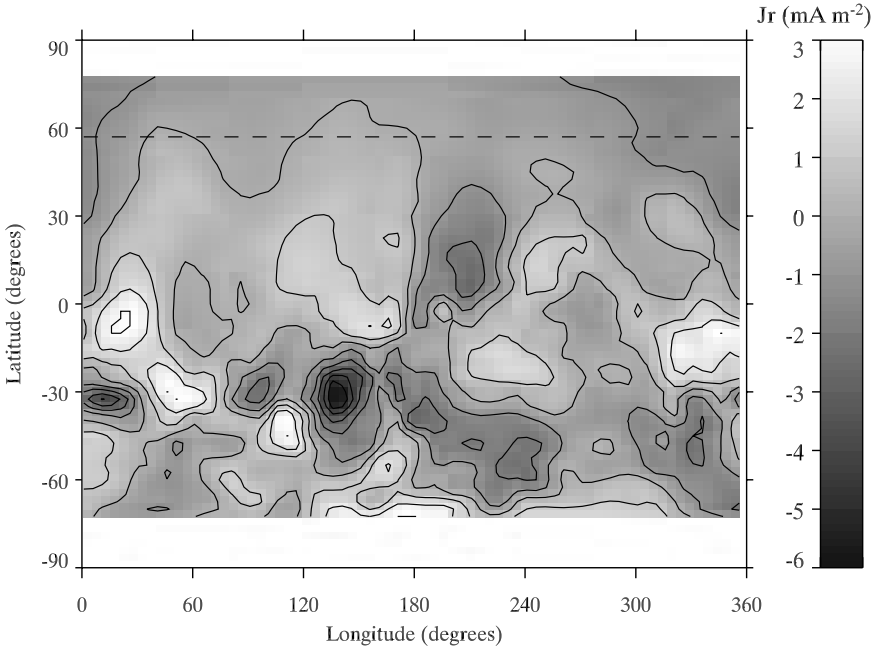

Fig. 12. Rectangular projection of the surface vertical current density $J_{r}\left(\mathrm{~mA} \mathrm{~m}^{-2}\right)$ according to the reconstructed surface magnetic field of 53 Cam. Numerical derivatives required to evaluate Eq. (12) could not be computed accurately for the magnetic field map at high rotational latitudes. Therefore the current density has not been calculated in the polar regions. The horizontal dashed line indicates the highest visible latitude corresponding to the adopted inclination of $53 \mathrm{Cam}$.

Equation (15) states that the net magnetic flux through the stellar surface must be null. Because of the $123^{\circ}$ inclination of the rotation axis of $53 \mathrm{Cam}$, part of the stellar surface is never presented to the observer or is observed close to the limb at only a few rotational phases. Although we can only evaluate Eq. (15) over the visible surface, by making certain judicious assumptions about the net flux in the invisible region we can estimate whether Eq. (15) is at least roughly satisfied.

Evaluating Eq. (15) we obtain a net flux over the visible surface of 53 Cam of $-1.63 \times 10^{20} \mathrm{kG} \mathrm{m}^{2}$, as compared to a net unsigned flux of $3.73 \times 10^{20} \mathrm{kG} \mathrm{m}^{2}$. This is a very large flux imbalance ( $44 \%$ of the total possible flux). Note that for test dipoles sampled on the same grid and analysed simultaneously with the same tools, we find a net flux imbalance of less than $0.2 \%$. Thus, it appears that the MDI map of 53 Cam does not satisfy Eq. (15).

Substantial flux imbalance is not a totally unexpected feature of our magnetic imaging based on the inversion technique with Tikhonov regularization and no global constraints on the large scale field structure. In general, due to the low signal-to-noise ratio of polarization spectra, incomplete phase coverage, visibility and projection effects there always exist areas on the stellar surface for which spectropolarimetric datasets cannot provide reliable information about the magnetic field structure. Analysis of the magnetic maps recovered in numerical experiments presented in Paper II shows that, although the typical imbalance of the net magnetic flux remains below $10-15 \%$ of the net unsigned flux, for some less favourable magnetic geometries it reaches magnitudes comparable to the one found for 53 Cam. 


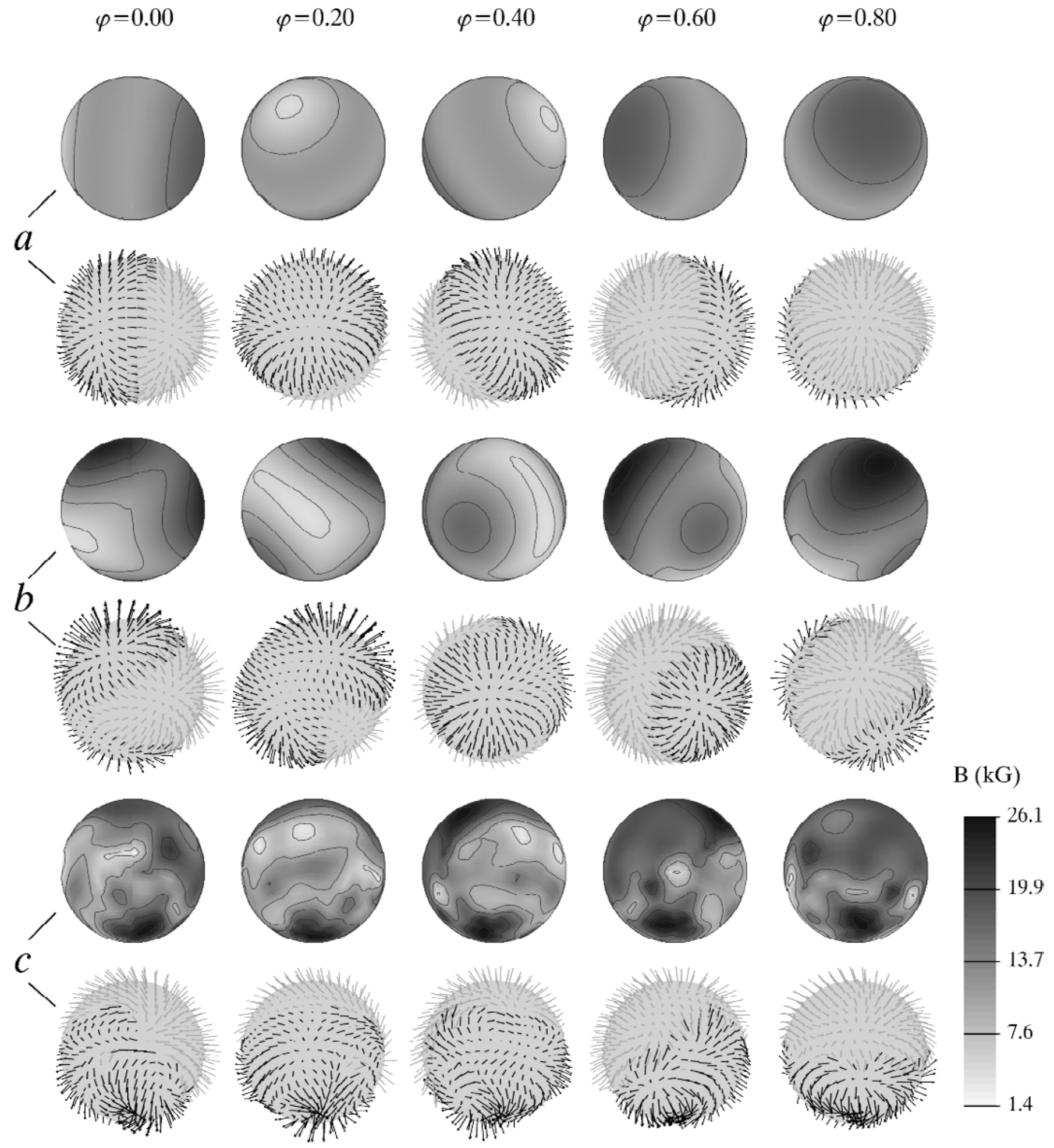

Fig. 13. The comparison of multipolar magnetic models of 53 Cam found by a) Landstreet (1988) and b) Bagnulo et al. (2001) with the average magnetic distribution derived in this study c). This figure is analogous to Fig. 5. In addition, the structures in the greyscale maps of the field strength (the upper panel in each pair of rows) are highlighted with contour lines plotted for the field strengths between 5 and $25 \mathrm{kG}$ with a step of $5 \mathrm{kG}$.

\subsection{Comparison with previous analyses of 53 Cam}

In Fig. 13 we compare the magnetic DI map derived for 53 Cam using INVERs10 with the results of the two most detailed recent investigations of the magnetic field morphology of this star (Landstreet 1988; Bagnulo et al. 2001). Both previous magnetic analyses assumed low-order multipolar parameterizations of the field topology and employed magnetic 
observables as well as some information from the Stokes $I$ line profiles (in Landstreet's 1988 study). The difference between multipolar models and the magnetic DI image is striking. Evidently, a much more complex field geometry emerges from the application of the DI technique and this complexity appears to be the key to a successful interpretation of the highresolution spectra in the four Stokes parameters. In contrast, the assumption of a low-order multipolar structure adopted in previous studies is clearly inadequate in the particular case of $53 \mathrm{Cam}$, since multipolar models fail to fit the Stokes IQUV spectra (see Bagnulo et al. 2001 and Sect. 5). Therefore, we conclude that parameters of the magnetic field topology of 53 Cam derived in multipolar analyses of this star characterize what appears to be an overly simplified description of a complex structure and, thus, must be interpreted with extreme caution.

\subsection{Implications for studies of magnetic fields in early-type stars}

We emphasize that our study questions the method of magnetic observables as a primary tool for revealing magnetic field morphologies of Ap stars. The example of 53 Cam demonstrates that a seemingly consistent interpretation of numerous high-quality measurements of the mean longitudinal field, magnetic field modulus and other observables does not necessarily lead to derivation of a realistic magnetic field model. Modelling of magnetic observables is by no means able to replace direct analysis of the high-resolution Stokes spectra and the latter should be preferred whenever possible.

Furthermore, we showed that even quite complex field distributions may result in fairly smooth phase curves of the magnetic observables. This suggests that a simple form of the variation of the mean longitudinal field and field modulus does not invariably imply a low-order multipolar magnetic topology, as was assumed in many investigations of the magnetic field geometries of early-type stars. Indeed, the parameter space in the range between the simplest first or second-order multipolar magnetic models and extremely complex tangled fields typical of solar-type stars remains largely unexplored. Given dramatic evidence of the field complexity revealed in $53 \mathrm{Cam}$, it would be extremely interesting to conduct a thorough mathematical analysis of the transformation between the stellar magnetic distributions and the Fourier coefficients of the phase curves of disk-integrated magnetic quantities in order to clarify the amount of information and the nature of constraints provided by the classical magnetic observables.

The detailed magnetic field map derived for 53 Cam opens exciting new possibilities for theoretical analyses of the basic magnetohydrodynamical processes responsible for generation and evolution of the global magnetic fields in Ap and related early-type stars. For example, our detection of an apparently significant contribution of the toroidal field components in the magnetic DI map of 53 Cam (see Sect. 7.1) is in agreement with theoretical expectations of Mestel \& Moss (1983), who argued that realistic stellar magnetic topologies must have a mixed poloidal-toroidal structure in the stellar interior in order to avoid instabilities and maintain a large-scale poloidal field on the stellar surface. We deduce that the system of electric currents in $53 \mathrm{Cam}$ is sufficiently extended to penetrate through the stellar atmosphere and give rise to the toroidal magnetic components observed in the line-forming region.

In general, the field complexity inferred in our study of $53 \mathrm{Cam}$ is not inconsistent with the theoretical expectations. It has been noted (e.g. Moss 2001) that there is no reason to expect the dynamo generated or fossil magnetic flux in earlytype stars to have an axis of symmetry and a simple multipolar configuration. In the process of stellar evolution magnetic configurations are further distorted by the magneto-centrifugal circulation in stellar envelopes (Moss 1990). Thus, taking into account the age of $53 \mathrm{Cam}$, we may suggest that this star had sufficient time to develop complex magnetic structures in the course of its evolution at the main sequence. Clearly, more detailed theoretical modelling is required to verify this hypothesis and to explain typical scales of the magnetic structures found on the surface of $53 \mathrm{Cam}$.

Our investigation of the magnetic field topology of $53 \mathrm{Cam}$ has demonstrated the very rich information content and the unique diagnostic potential of high-resolution stellar Stokes spectra. Obviously, magnetic Doppler mapping has to be applied to spectropolarimetric observations of other Ap stars in order to characterize their magnetic morphologies at the same level of detail that we achieved for $53 \mathrm{Cam}$. The most important question to be addressed in future studies is whether the complex field structure found in 53 Cam represents a typical example or a very special case of the field organization in early-type stars.

Acknowledgements. This work was supported by the Swedish National Research Council and has been partly funded by the Natural Sciences and Engineering Research Council of Canada through grants to GAW, JDL and TAAS. We would like to thank the Knut and Alice Wallenberg Foundation for contributing to the state-of-the-art computing facilities at the Institute of Astronomy and Space Physics in Uppsala. We are also grateful to Eric Stempels and Paul Barklem for their assistance in obtaining and reducing the INT/MuSiCoS spectrum of 53 Cam.

This research has made extensive use of the SIMBAD database, operated at CDS, Strasbourg, France.

\section{References}

Abramenko, V. I., Gopasyuk, S. I., \& Ogir, M. B. 1990, in Solar Photosphere: Structure, Convection and Magnetic Fields, ed. J. O. Stenflo (Dordrecht: Kluwer), 267

Alecian, G., \& Stift, M. J. 2002, A\&A, 387, 271

Babcock, H. W. 1958, ApJ, 128, 228

Babcock, H. W. 1960, ApJ, 132, 521

Bagnulo, S., Landolfi, M., \& Landi Degl'Innocenti, M. 1999, A\&A, 343,865

Bagnulo, S., \& Wade, G. A. 2001, Magnetic Fields Across the Hertzsprung-Russell Diagram, ed. G. Mathys, S. K. Solanki, \& D. T. Wickramasinghe, ASP Conf. Ser., 248, 325

Bagnulo, S., Wade, G. A., Donati, J.-F., et al. 2001, A\&A, 369, 889

Barklem, P. B., Stempels, H. C., Allende Prieto, C., et al. 2002, A\&A, 385,951 
Baudrand, J., \& Böhm, T. 1992, A\&A, 259, 711

Borra, E. F., \& Landstreet, J. D. 1977, ApJ, 212, 141

Carrier, F., North, P., Udry, S., \& Babel, J. 2002, A\&A, 394, 151

Dolk, L., Wahlgren, G. M., Lundberg, H., et al. 2002, A\&A, 385, 111

Donati, J.-F., Semel, M., Carter, B. D., Rees, D. E., \& Cameron, A. C. 1997, MNRAS, 291, 658

Donati, J.-F. 1999, MNRAS, 302, 457

Donati, J.-F., Catala, C., Wade, G. A., et al. 1999, A\&AS, 134, 149

ESA 1997, The Hipparcos Catalogue, ESA SP-1200

Flower, P. J. 1996, ApJ, 469, 355

Gray, D. F. 1992, The Observation and Analysis of Stellar Photospheres (Cambridge University Press)

Hussain, G. A. J., Donati, J.-F., Cameron, A. C., \& Barnes J. 2000, MNRAS, 318, 961

Hussain, G. A. J., Jardine, M., \& Cameron, A. C. 2001, MNRAS, 322, 681

Hill, G. M., Bohlender, D. A., Landstreet, J. D., et al. 1998, MNRAS, 297, 236

Hubrig, S., North, P., \& Mathys, G. 2000, ApJ, 539, 352

Huchra, J. 1972, ApJ, 174, 435

Kemp, J. C., \& Wolstencroft, R. D. 1974, MNRAS, 166, 1

Khokhlova, V. L., Vasilchenko, D. V., Stepanov, V. V., \& Romanyuk I. I. 2000, Astron. Lett., 26, 177

Kochukhov, O., \& Piskunov, N. 2002, A\&A, 388, 868 (Paper II)

Kochukhov, O., Piskunov, N., Ilyin, I., Ilyina, S., \& Tuominen, I. 2002, A\&A, 389, 420 (Paper III)

Kupka, F., Piskunov, N., Ryabchikova, T. A., Stempels, H. C., \& Weiss, W. W. 1999, A\&AS, 138, 119

Kurucz, R. 1993, CD-ROM No. 13, Smithsonian Astrophys. Obs.

Kurucz, R. L., Furenlid, I., Brault, J., \& Testerman, L. 1984, NSO Atlas No. 1: Solar Flux Atlas from 296 to 1300 nm, Sunspot, NSO

Kuschnig, R., Ryabchikova, T. A., Piskunov, N. E., Weiss, W. W., \& Gelbmann, M. J. 1999, A\&A, 348, 924

Landi Degl'Innocenti, M., Calamai, G., Landi Degl'Innocenti, E., \& Patriarchi, P. 1981, ApJ, 249, 228

Landolfi, M., Bagnulo, S., \& Landi Degl'Innocenti, M. 1998, A\&A, 338,111

Landstreet, J. D. 1988, ApJ, 329, 927

Landstreet, J. D., Barker, P. K., Bohlender, D. A., \& Jewison, M. S. 1989, ApJ, 344, 876

Landstreet, J. D. 1992, A\&ARv, 4, 35

Leroy, J. L., Landolfi, M., Landi Degl'Innocenti, M., et al. 1995, A\&A, 301, 797
Lucke, P. B. 1978, A\&A, 64, 367

Lutz, T. E., \& Kelker, D. H. 1973, PASP, 85, 573

Martin, C., \& Mignard, F. 1998, A\&A, 330, 585

Mathys, G. 1989, Fund. Cosmic Phys., 13, 143

Mathys, G., Hubrig, S., Landstreet, J. D., Lanz, T., \& Manfroid, J. 1997, A\&AS, 123, 353

McAlister, H. A., Hendry, E. M., Hartkopf, W. I., Campbell, B. G., \& Fekel, F. C. 1983, ApJS, 51, 309

Mestel, L., \& Moss, D. L. 1983, MNRAS, 204, 575

Metcalf, T. R., Canfield, R. C., Hudson, H. S., Mickey, D. L., \& Wülser, J.-P. 1994, ApJ, 428, 860

Michaud, G. 1970, ApJ, 160, 641

Michaud, G., Charland, Y., \& Megessier, C. 1981, A\&A, 103, 244

Moss, D. 1990, MNRAS, 244, 272

Moss, D. 2001, in Magnetic Fields Across the Hertzsprung-Russell Diagram, ed. G. Mathys, S. K. Solanki, \& D. T. Wickramasinghe, ASP Conf. Ser., 248, 305

Peterson, D. M. 1969, Smithsonian Astrophys. Obs. Spec. Rep., 244

Piskunov, N., \& Kochukhov, O. 2002, A\&A, 381, 736 (Paper I)

Preston, G. W. 1972, ApJ, 175, 465

Pyper, D. M., \& Adelman, S. J. 1983, A\&AS, 51, 365

Ryabchikova, T., Piskunov, N., Kochukhov, O., et al. 2002, A\&A, 384, 545

Schaller, G., Schaerer, D., Meynet, G., \& Maeder, A. 1992, A\&AS, 96, 269

Semel, M., Donati, J.-F., \& Rees, D. E. 1993, A\&A, 278, 231

Stehlé, C. 1994, A\&AS, 104, 509

Stibbs, D. W. N. 1950, MNRAS, 110, 395

Tokovinin, A. 1992, in Complementary Approaches to Double and Multiple Star Research, ed. H. A. McAlister, \& W. I. Hartkopf, ASP Conf. Ser., 32, 573

Vidal, C. R., Cooper, J., \& Smith, E. W. 1973, ApJS, 25, 37

Wade, G. A., Donati, J.-F., Landstreet, J. D., \& Shorlin, S. L. S. 2000a, MNRAS, 313, 823

Wade, G. A., Donati, J.-F., Landstreet, J. D., \& Shorlin, S. L. S. 2000b, MNRAS, 313, 851

Wade, G. A., Bagnulo, S., Kochukhov, O., et al. 2001, A\&A, 374, 265

Wade, G. A. 2002, A Peculiar Newsletter, 38, 3

Wade, G. A., LeBlanc, F., Ryabchikova, T., \& Kudryavtsev, D. 2003, in Modelling of Stellar Atmospheres, IAU Symp. 210, ed. N. Piskunov, W. W. Weiss, \& D. F. Gray, ASP Conf. Ser., in press

Wang, J. 1993, in The magnetic and velocity fields of solar active regions, ed. H. Zirin, G. Ai, \& H. Wang, ASP Conf. Ser., 46, 425 